\title{
Aerosol Particle Collector Design Study
}

\author{
Richard A. Dimenna and Si Young Lee
}

September 2007

Washington Savannah River Company

Savannah River National Laboratory

Aiken, SC 29808

Prepared for the U.S. Department of Energy

Under Contract No. DE-AC09-96SR18500

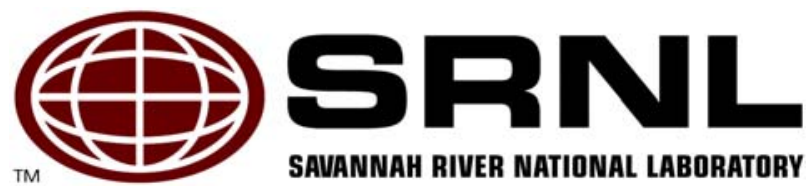




\section{DISCLAIMER}

This report was prepared by Washington Savannah River Company (WSRC) for the United States Department of Energy under Contract No. DE-AC09-96SR18500 and is an account of work performed under that contract. Neither the United States Department of Energy, nor WSRC, nor any of their employees makes any warranty, expressed or implied, or assumes any legal liability or responsibility for the accuracy, completeness, or usefulness, of any information, apparatus, or product or process disclosed herein or represents that its use will not infringe privately owned rights. Reference herein to any specific commercial product, process, or service by trademark, name, manufacturer or otherwise does not necessarily constitute or imply endorsement, recommendation, or favoring of same by WSRC or by the United States Government or any agency thereof. The views and opinions of the authors expressed herein do not necessarily state or reflect those of the United States Government or any agency thereof.

\section{Printed in the United States of America}

Prepared For

U.S. Department of Energy 
Keywords: Particle collection,

Aerosol flow, Turbulent flow,

Computational Fluid Dynamics

\section{Aerosol Particle Collector Design Study}

Richard A. Dimenna and Si Young Lee

September 2007

Washington Savannah River Company

Savannah River National Laboratory

Aiken, SC 29808

Prepared for the U.S. Department of Energy

Under Contract No. DE-AC09-96SR18500

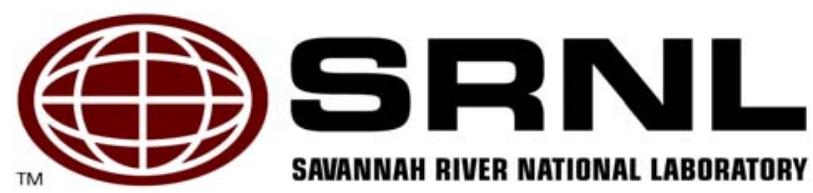




\section{Review and Approvals}

R. A. Dimenna, Author Date

Eng. Modeling and Simulation Group, SRNL

S. Y. Lee, Coauthor Date

Eng. Modeling and Simulation Group, SRNL

B. B. Anderson, Customer Reviewer Date

Nonproliferation Tech. Section, SRNL

M. V. Gregory, Technical Reviewer Date

Eng. Modeling and Simulation Group, SRNL

S. J. Hensel, Manager Date

Eng. Modeling and Simulation Group, SRNL 


\section{Table of Contents}

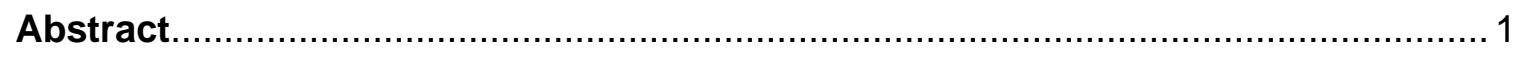

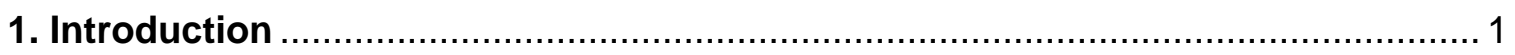

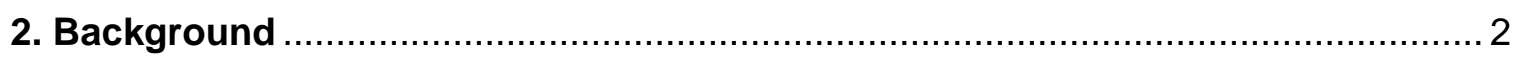

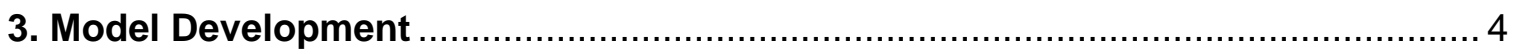

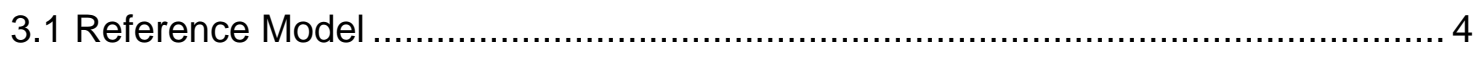

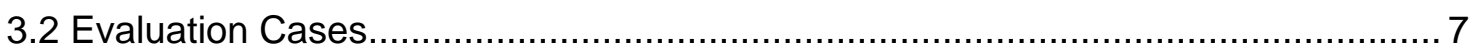

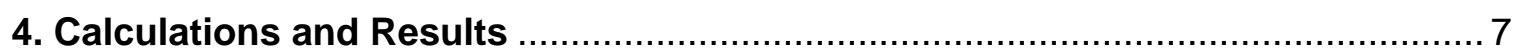

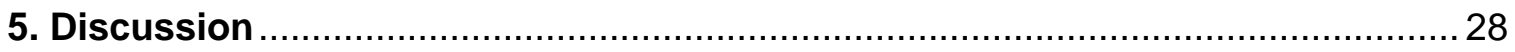

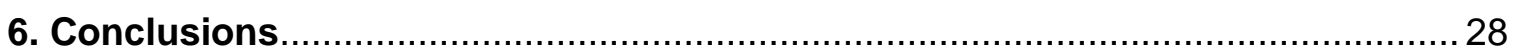

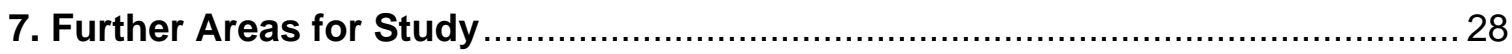

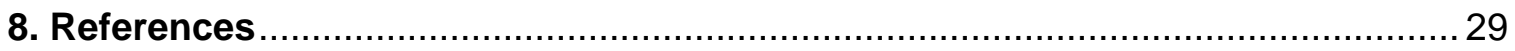

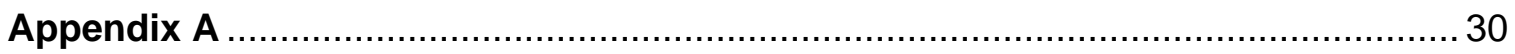

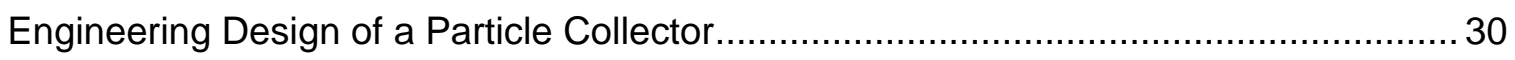




\section{List of Figures}

Figure 1. Cross-sectional view of the reference design during the particle collection

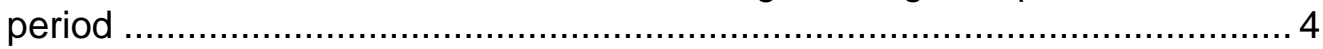

Figure 2. Reference design for particle collector (standing on its side). ...................... 5

Figure 3. Cross-sectional view of the modeling region during the particle collection

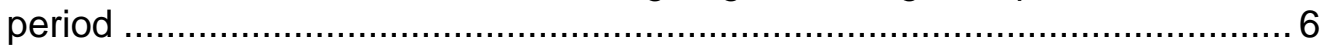

Figure 4. Collection efficiency as function of applied field strength for $1-\mu \mathrm{m}$ particle at

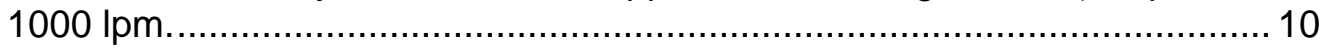

Figure 5. Collection efficiency vs. dielectric constant for 8-in collection length............... 11

Figure 6. Collection efficiencies vs. particle size for two dielectric constants and the

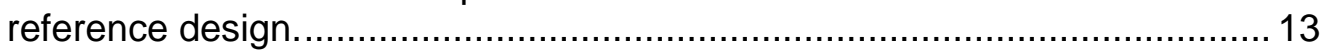

Figure 7. Collection efficiency vs. dielectric constant for two different collection lengths.13

Figure 8. SRNL recommended collector geometry based on $1000 \mathrm{lpm}$ flow rate and no design restrictions.

Figure 9. Comparison of collection efficiency between the two collectors for 1- $\mu \mathrm{m}$ particle under 1000 liter/min .................................................................. 17

Figure 10. Comparison of velocity contours between the two collectors for 1- $\mu \mathrm{m}$ particle

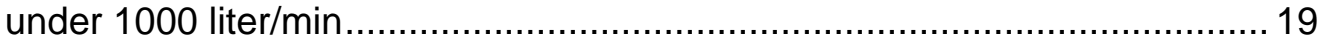

Figure 11. CFD models for the reference and L-type collectors ………………….... 20

Figure 12. Comparison of velocity contours for the reference and L-type collectors ..... 21

Figure 13. Comparison of the turbulent intensities for the reference and L-type collectors

Figure 14. Velocity contour for 1.8-in separation gap distance for 2:1 transition region 24

Figure 15. Comparison of vertical velocity components for the collectors with different wall surface conditions 25

Figure 16. Comparison of flow patterns for the collectors with different wall surface conditions 26

Figure 17. Collection plate with 0.3 -in flow obstruction near the entrance of collector region 


\section{List of Tables}

Table 1. Comparison of collection efficiencies for various sampling flow rates for the reference collector design shown in Fig. 2. ....................................... 9

Table 2. Collection efficiency of the reference design collector for a 1- $\mu \mathrm{m}$ particle and various dielectric constants.

Table 3. Results of collection efficiency for various particle sizes under the reference design of collector (dielectric constant $=4.3$ (quartz))

Table 4. Results of collection efficiency for various particle sizes for the reference design collector (dielectric constant $=10$ )

Table 5. Results of collection efficiency for various designs (dielectric constant $=4.3$ (quartz))

Table 6. Power requirement comparison for different inlet geometries to the collection region 


\section{Abstract}

A computational evaluation of a particle collector design was performed to evaluate the behavior of aerosol particles in a fast flowing gas stream. The objective of the work was to improve the collection efficiency of the device while maintaining a minimum specified air throughput, nominal collector size, and minimal power requirements. The impact of a range of parameters was considered subject to constraints on gas flow rate, overall collector dimensions, and power limitations. Potential improvements were identified, some of which have already been implemented. Other more complex changes were identified and are described here for further consideration. In addition, fruitful areas for further study are proposed.

\section{Introduction}

An aerosol is a two-phase medium which can be composed of solid particles or liquid drops dispersed in gas phase. In certain groups of aerosol applications, charging the particles can be required to change their electrohydrodynamic properties to enhance the efficiency of technological processes such as electrostatic surface coating or painting, particle separation, electrostatic scrubbing, electrostatic precipitation, crop spraying, or mass spectroscopy. In the application described here, charging the particles allows the use of an electrostatic force to drive particles to the collection surface and remove them from the free stream.

The work described in this report was to evaluate the behavior of aerosol particles in a fast flowing gas stream with the objective of improving the collection efficiency of the collector. The impact of a range of parameters was considered, although constraints such as gas flow rate, overall collector dimensions, and power limitations restricted flexibility in changing some. Nonetheless, potential improvements were identified, some of which have already been implemented. Other more complex changes are described here for further consideration. In addition, fruitful areas for further study are proposed.

The approach described here groups the phenomena investigated into two principal categories: those that affect the force acting on the aerosol particles, and those that affect the time available to move a particle from the free stream to the collection plate. Examples of the former include parameters that affect particle charge such as size, corona shape, proximity of the corona discharge region to the collection region, humidity, etc. Examples of the latter are flow rate, collector geometry and inlet flow shaping. Issues such as free stream turbulence, collector plate geometry, and boundary layer effects have an impact on both categories. The work being reported here is an initial look at many of these phenomena.

The work described in this report is based on a nominal gas flow rate of 1000 liters/min (Ipm) through the collector. Since the time that this work was performed, changes to the design have been made to double the nominal flow rate to $2000 \mathrm{lpm}$. The general findings reported here are applicable to both flow rates, but the specific calculated results such as pressure drop, etc. apply only to the flow rates stated in the tables. There is no work reported here specifically for the 2000 lpm configuration. 


\section{Background}

As air flows through a duct, particles suspended in the air may tend to settle out and stick to the walls of the duct. The parameters that affect how many particles fall out of suspension are the velocity of the air passing through the duct, the surface area $\left(A_{c}\right)$ of the duct available for particle collection, and the settling velocity $\left(V_{s}\right)$ of the suspended particles.

The extent to which these parameters influence particle deposition depends in part on whether the air flow is laminar or turbulent. Laminar flow does not have the random velocity fluctuations that significantly enhance the degree of mixing associated with turbulent flow. The impact of turbulent mixing on particle motion can have a strong effect on the rate of particle deposition on the collection surface. In addition, if the aerosol particles are charged and an electric field is applied in the collector, the electrostatic force is dominant in the collector. In this case, the collector is called an electrostatic precipitator (ESP) and the settling velocity is replaced with the terminal electrostatic velocity, $V_{\mathrm{TE}}$. Several models have been created in an attempt to accurately describe the collection efficiency of an electrostatic precipitator. Many of these are discussed in the Appendix to this report.

Several physical factors influence the collection efficiency of the ESP. The successful engineering of an ESP depends ultimately on how efficiently particles can be made to contact the collector surface and how efficiently they can be made to stay there. Large collector areas and low volumetric flow rates help to increase efficiency because they increase the amount of time that the collector has to capture particles. If the maximum allowable collector size and minimum allowable flow rate have been reached and the collector efficiency is not as high as desired, then stronger forces must be applied to get the particles to the collection wall quicker. This is accomplished in an ESP by ensuring that the particles to be collected acquire a maximum number of fundamental charges and by imposing the strongest possible electric field.

General Collector Behavior The physics of the particle collector center largely around a time-of-flight analysis for the particles. The particles are dragged with the entering flow at essentially the same speed as the gas flow. At the same time, they have a drift velocity in the transverse direction which is determined by the forces on the particle, principally an electrostatic force resulting from the imposed electric field acting on the charge carried by the particle. The gas flow determines the time during which the particle is exposed to the electric field, and the geometry of the collector determines whether that time is sufficient for the particle to reach the collector surface. A more complex parameter that is both harder to characterize and harder to control than the collector geometry or the imposed electric field is the turbulence intensity within the collector. Neither the influence of the turbulent eddies on particle motion nor the behavior of the particle as it reaches the turbulent boundary layer at the collection surface has been characterized in this study.

The parameters that are addressed in this study include:

- Geometry of the collector: The principal concern with collector geometry is the proximity of the particles to the collection surface as they enter the collector region. For a fixed collection length, closer proximity should translate to better collection efficiency. This behavior was explored by modifying the collection region to force the particles closer to the collection plate, as well as by modifying the inlet region to take advantage 
of particle inertia to move them closer to the collection plate as they enter the collection region. An additional geometric modification was explored by modifying the outlet geometry to smooth the flow and reduce the total pressure drop through the collector. This resulted in a greater flow rate through the device for the same electrical power to the exhaust fans.

- Electric field strength: A stronger electric field should result in a greater force on the particle and a shorter time required to attract it to the collection surface.

- Particle charge: Particle size, humidity of the surrounding atmosphere, and the strength and shape of the corona region probably impact the total charge that can be carried on a particle. This in turn affects the electrostatic force attracting the particle to the collection surface in the same manner as the electric field strength.

- Collection surface: The flow characteristics of the collection surface, in particular the roughness of the surface, are thought to impact the development and thickness of the boundary layer and the degree of turbulence intensity in the flow near the surface. The impact of this behavior on particle collection is not well understood, nor has it been addressed in this study. Nonetheless, it is thought to be potentially important and worth exploring in future work.

Collection Efficiency Special attention was paid to the charge carried by the individual particles because of the importance of the electrostatic force.

When charged particles pass through a strong electric field, they are attracted toward the electrode of opposite polarity. The expression for determining the efficiency of particle collection can be obtained as shown in the literature correlation for the flat plate (Deutsch, 1922), assuming that particles stick to the collection plate if they touch it and do not reenter the fluid region.

The fraction of particles removed during the collection period is equal to the ratio of the thin collection region to total cross-sectional area of the duct as shown in Figure 1. That is

$\left(\frac{d N}{N}\right)=-\frac{W v_{p} d t}{D W}=-\frac{v_{p} d t}{D}$

By integrating Eq. (1) between the limits of the number of particles entering the collector, $N_{o}$, to the number $N$ exiting after a time t, the ratio of exiting to entering particles during the particle residence time $\tau$ becomes

$\left(\frac{N}{N_{o}}\right)=\exp \left(-\frac{v_{p} \tau}{D}\right)$

This expression assumes that the particles are continuously and homogeneously mixed in the collector duct as they pass through the collection region. The collection efficiency $\eta$ for a wire and plate system is then

$\eta=\frac{N_{o}-N}{N_{o}}=1.0-\exp \left(-\frac{v_{p} L_{c}}{D v_{g}}\right)$.

In Eq (3) $N_{o}$ is the number of suspended particles entering the system, $N$ the number escaping the system, $v_{p}$ the drift velocity of the particle, $L_{c}$ the length of the collector, $D$ the distance between electrodes, and $v_{g}$ the air velocity. The drift velocity of the particle $v_{p}$ is given by the force balance between the Stoke's drag and electrical force. That is 
$v_{p}=\left(\frac{V_{E}}{D}\right)\left(\frac{n e}{3 \pi \mu d_{p}}\right)$

where $V_{E}$ is the electrical voltage between the electrodes, $n$ the number of electrons on the particle surface, e electron charge, $\mu$ the air viscosity, and $d_{p}$ the particle diameter.

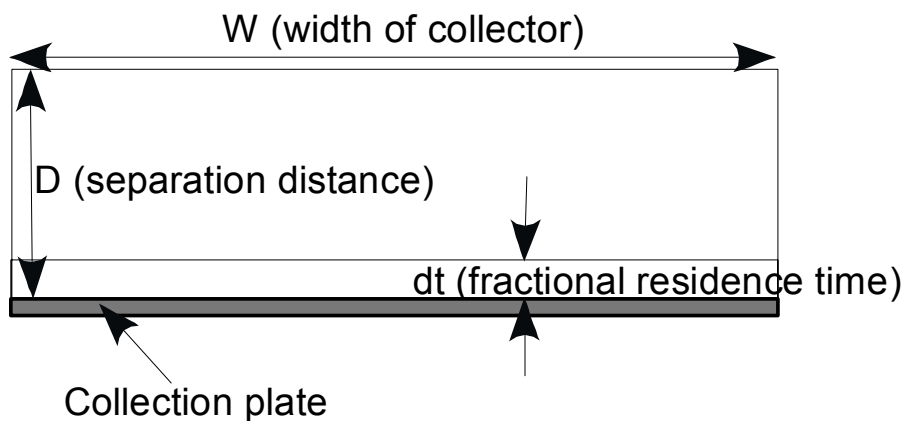

Figure 1. Cross-sectional view of the reference design during the particle collection period

The electrostatic force on a particle is directly proportional to the charge carried by the particle. Particle charge $q$ is a function of particle size, and can be described by Cochet's charge equation (Kim et al., 2001),

$q=4 \pi \varepsilon_{0} E r^{2}\left[\left(1+\frac{\lambda_{i}}{r}\right)^{2}+\left(\frac{2}{1+\left(\frac{\lambda_{i}}{r}\right)}\right)\left(\frac{\kappa-1}{\kappa+2}\right)\right]$,

where $\varepsilon_{0}$ is the permittivity of free space, $E$ the local electric field strength, $\lambda_{i}$ the ionic mean free path, $r$ the particle radius, and $\kappa$ the dielectric constant of particles. The ability to maximize the charge on a particle is a key consideration in the design of the collector.

\section{Model Development}

\subsection{Reference Model}

The models used to test the physical parameters described above were all modifications from a base model of an initial apparatus referred to here as the reference design. It is shown standing on its side in Figure 2 as a rectangular geometry 4 inches wide and 1 inch high with a collection plate 8 inches long and a corona wire 1.875 inches upstream of the collection region. The discharge region for the reference design has already incorporated changes recommended from an initial inspection of the apparatus (removing an L-shaped bend at the outlet of the device). The abrupt change in flow area at the outlet of the apparatus still exists as shown in Figure 2. 
Since the time of this original work, modifications have been made to the geometry to support a higher flow rate, $2000 \mathrm{lpm}$. This includes significant changes to the outlet geometry. These changes have not yet been addressed computationally, but they include smoothing the transition between the collection region and the outlet expansion region.

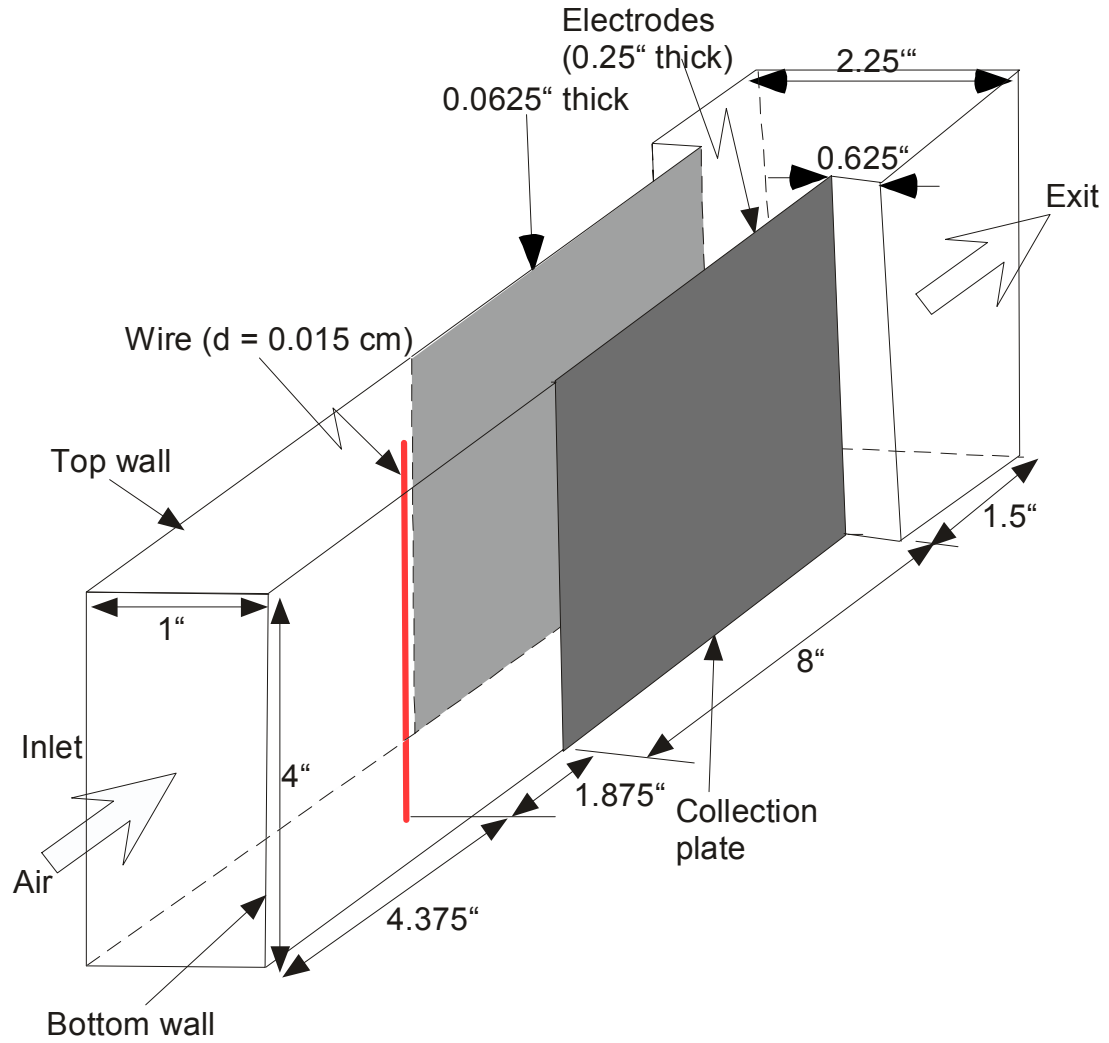

Figure 2. Reference design for particle collector (standing on its side).

A Fluent model of the reference design was developed and run using nominal boundary conditions to test the model performance against measured performance. These results will be described in detail in the next section. The model of the reference design consisted of approximately 200,000 nodes. The boundary conditions and typical physical parameters were as follows:

Inlet flow boundary: mass flow with a uniform velocity calculated to provide the desired flow rate, typically $1000 \mathrm{lpm}$.

Outlet flow boundary: constant pressure.

Solid surfaces: no-slip.

Initial conditions: Since the calculations were all steady-state solutions, initial conditions were not required, except perhaps to speed convergence.

Air properties: $\quad$ typical for $20^{\circ} \mathrm{C}$ 
Particle size: $\quad$ typically $1 \mu \mathrm{m}$ with sensitivity calculations for smaller sizes

Electric field strength: nominally $15000 \mathrm{~V} /$ in $(5900 \mathrm{~V} / \mathrm{cm})$

Particle charge: nominally assume saturated charge conditions

Surface roughness: $1 \mu \mathrm{m}$

Energy parameters: the energy equation was not included in the analysis, therefore adiabatic boundaries and isothermal flow

While sensitivity to the air flow rate through the apparatus was calculated, the nominal flow rate of $1000 \mathrm{lpm}$ was taken as a physical requirement throughout the analysis. The calculated sensitivity to changes in the flow rate were used more to test the model to ensure it was delivering qualitatively acceptable results than to search for a higher collector efficiency as a function of air flow rate.

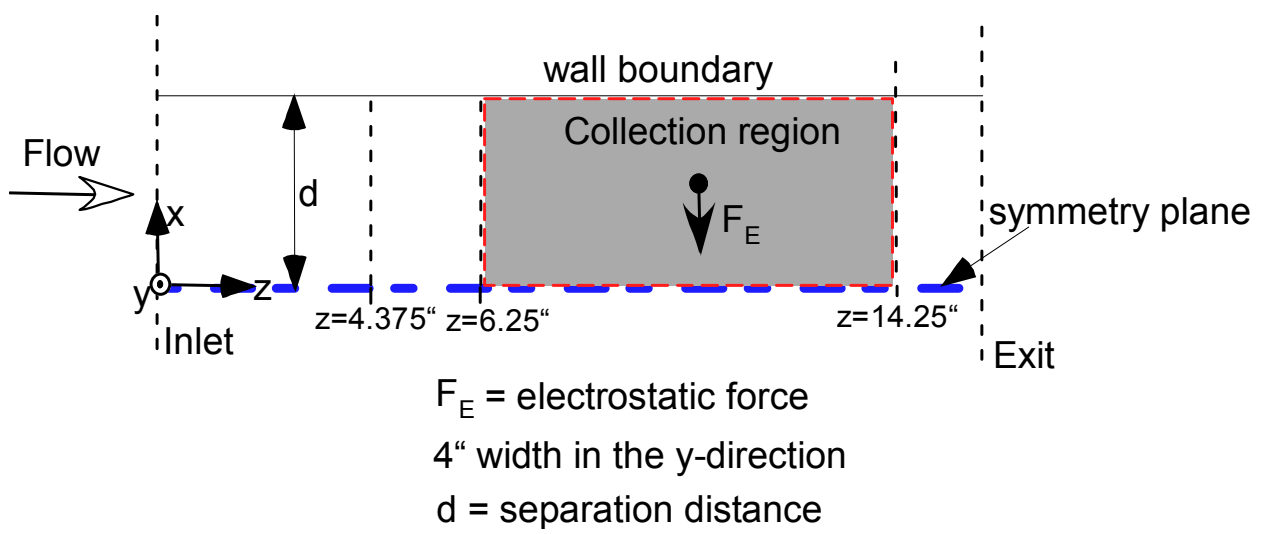

Figure 3. Cross-sectional view of the modeling region during the particle collection period

The electrostatic force, $F_{E}$, shown in Fig. 3 is modeled in Fluent using a "user defined file" (UDF) to define an additional body force term in the flow calculations (see the dashed line in red in Fig. 3) [Cochet, 1961, Li and Ahmadi, 1993].

$F_{E}=q E-\frac{q^{2}}{16 \pi \varepsilon_{0} x^{2}}+\frac{q E d^{3}}{16 x^{3}}-\frac{3 \pi \varepsilon_{0} d^{6} E^{2}}{128 x^{4}}$

and

$E=\frac{V}{d}$

where $d$ is a separation distance between the conducting plates across which the potential difference $\mathrm{V}$ is applied. 
The first term in Eq. 6 is the Coulomb force due to the applied electric field. The second term on the right-hand side is the image force corresponding to the force exerted by an image charge of $-q$ at position $-x$ from the surface. The third term is the dielectrophoretic force on the induced dipole due to the gradient of the field from the image charge. The last term is the dipole-dipole force due to the interaction of the induced dipole and its image. The image and dipole-dipole forces are always directed toward the surface, while the Coulomb and dielectrophoretic forces can be either toward or away from the wall. In this study, Coulomb and image forces are included in the analysis since the scoping calculations show that the other forces are negligibly small.

\subsection{Evaluation Cases}

The cases developed with specific models were:

1. Geometry variations
a. Center plate
b. L-shaped inlet
c. Curved inlet
d. Smoothed outlet
e. Collection plate single obstruction
f. Collection plate multiple obstructions

2. Boundary condition modifications
a. Flow rate

3. Electrostatic force modifications
a. Particle charge
b. Imposed electric field strength
c. Humidity (particle charge)
d. Dielectric constant (related to humidity, particle material)

The results from all of these modifications will be discussed in the next section, Calculations and Results, along with a discussion of the particular characteristics of the model or the device being tested.

\section{Calculations and Results}

The calculated results are presented in two parts. First is a discussion of the base case calculations for the reference design. These results are compared to literature correlations to establish the validity of both the reference design itself and the computational method used to evaluate its performance. The calculations of all the remaining cases are then compared to the results from the reference design. In 
addition, comparisons are made to the literature where appropriate, and in particular to results and behavior described in Hinds (1999). All calculated results described here are based on steady-state calculations with the Fluent model representing either the base case (reference design) or modifications from that design as described for the particular sensitivity cases.

The calculations made for this study can be arranged into two main groups in addition to the base case. In Group 1 are sensitivity analyses designed to characterize the impact of changing the attractive force between the charged particles and the collection plate. These include variations in the length of the collection plate, the particle size and dielectric constant, and the field strength imposed in the collector region. The humidity of the air entering the collector fell into this group because of its impact on the charge accumulated by the particles. The Group 1 analyses assume the height of the channel in the collector is fixed at 1 inch as specified for the reference design.

Group 2 analyses addressed options for reducing the distance between the particles and the collection plate so that the particles had a shorter distance to travel to be captured. The simplest (and most effective) case was simply to add a thin collection plate along the centerline of the collector and adjust the electric field so it attracted the particles to the center collection plate in both halves of the collector region (shown later in Figure 8). For this initial analysis, no consideration was given to the means by which particles could be collected on either side of the collection plate and removed easily. Such matters, while important, were considered secondary to the theoretical performance of the design change. Other changes that were tested included various geometrical changes to the inlet region designed to move the particles closer to the collection plate mechanically before they entered the collection region. While some geometrical modifications were identified that were successful in doing this, the changes were not as effective as adding a center plate, largely because the air in which the particles were contained also moved closer to the collection plate, in effect reducing the cross-sectional area of the collector and effectively increasing the axial velocity consistent with flow through a smaller duct.

\section{Base Case}

The results of the reference case are shown in Table 1. The operating geometry and conditions for the Reference Design collector include:

1. A collector cross-section 4 inches wide by 1 inch high.

2. A collector plate 8 inches long. This will be referred to as the collector region.

3. $1000 \mathrm{l} / \mathrm{min}$ air flow rate

4. A straight inlet region with a centered corona wire 1.875 inches upstream of the collector region.

5. An electric potential of 15000 volts on the collector plate relative to the reference plate that defined the opposite surface of the collector region. 
Table 1. Comparison of collection efficiencies for various sampling flow rates for the reference collector design shown in Fig. 2.

\begin{tabular}{|c|c|c|c|}
\hline \multirow{2}{*}{$\begin{array}{c}\text { Sampling flowrate } \\
\text { conditions }\end{array}$} & \multirow{2}{*}{$\begin{array}{l}\text { Particle } \\
\text { size }(\mu \mathrm{m})\end{array}$} & \multicolumn{2}{|c|}{ Efficiency } \\
\hline & & Modeling predictions & Correlations (Eq . (3)) \\
\hline $1000 \mathrm{lpm}$ & 1 & $23 \%$ & $19 \%$ \\
\hline 600 Ipm & 1 & $37 \%$ & $30 \%$ \\
\hline 600 Ipm & 0.3 & $13 \%$ & $10 \%$ \\
\hline
\end{tabular}

\section{Group 1 Calculations - Fixed collector geometry}

The Group 1 calculations focus on parameters that affect the electrostatic force on the charged particles or the impact of that force on the collector efficiency. This includes the electric field strength, the amount of charge carried by each particle, and the length of the collector, since increasing the collector length provides more time to collect the particles but does not change the flow pattern in any way that would impact the transverse motion of the particles. Each of these parameters will be discussed in this section.

Electric field strength The electrostatic force attracting the charged particle to the collection surface is the only significant force acting transverse to the principal flow direction. The only other constant force acting on the particles is gravity, and it is far smaller than the electrostatic force.

For a fixed geometry in the collector region and a given charge per particle, it is expected that a stronger electric field would result in a stronger force on the particles, and therefore a larger collection efficiency. This result is shown in Figure 4 and compared to a similar sensitivity as calculated by the Deutsch-Anderson correlation. The CFD results indicate that for a sufficiently high field strength, essentially all the particles entering the collection region are captured at the collection plate. The DeutschAnderson correlation, on the other hand, shows an increase in collection efficiency to about $50 \%$ at the maximum field strength tested. The reason for this difference in behavior is the fundamental nature of the two calculations. The Deutsch-Anderson equation describes a particle distribution that remains uniform throughout the entire cross-section of the collector [3]. This results in the exponential form of the equation as particles are removed from that differential layer close to the surface and the rest are immediately and uniformly redistributed. The Fluent calculation, on the other hand, imposes a net force on the particles and attracts them to the collection surface in much the same way as would be expected in a laminar flow. The impact of turbulence on particle motion might make the Deutsch-Anderson approach reasonable, but this has not been evaluated in this study. 


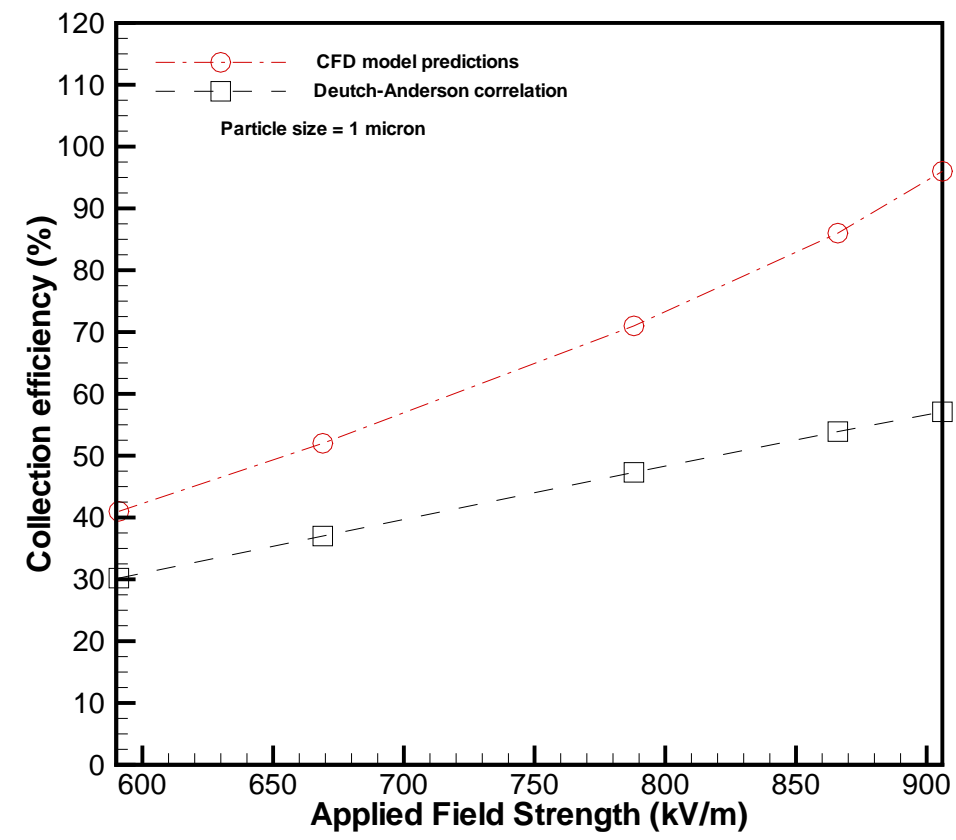

Figure 4. Collection efficiency as function of applied field strength for $1-\mu \mathrm{m}$ particle at $1000 \mathrm{lpm}$.

Dielectric constant The dielectric constant was modified to evaluate the impact on the saturation charge collected by a given particle. This in turn was used to modify the electrostatic force with which the particle was attracted to the collection surface. Table 2 shows the comparative results for three different particle materials, all based on a $1 \mu \mathrm{m}$ particle. The collection efficiency was determined from a calculation of the particle behavior in the reference geometry computational model. Figure 5 shows the impact of the dielectric constant for two different values of the constant.

Particle size Particle size affects the ability of a particle to accumulate charge, and therefore, the electrostatic force on the particle, much the same as the dielectric constant affects the particle charge. Tables 3 and 4 show the impact of particle size on both the saturation charge and the corresponding collection efficiency for the reference geometry model. The results are plotted in Figure 6. 
Table 2. Collection efficiency of the reference design collector for a 1- $\mu \mathrm{m}$ particle and various dielectric constants.

\begin{tabular}{|c|c|c|}
\hline Dielectric constant & $\begin{array}{c}\text { Number of electron charges } \\
\text { (based on 15KV/inch) }\end{array}$ & Collection efficiency (\%) \\
\hline 4.3 (quartz) & 237 & 23 \\
\hline 10.0 & 276 & 26 \\
\hline 80.0 (water) & 313 & 29 \\
\hline
\end{tabular}

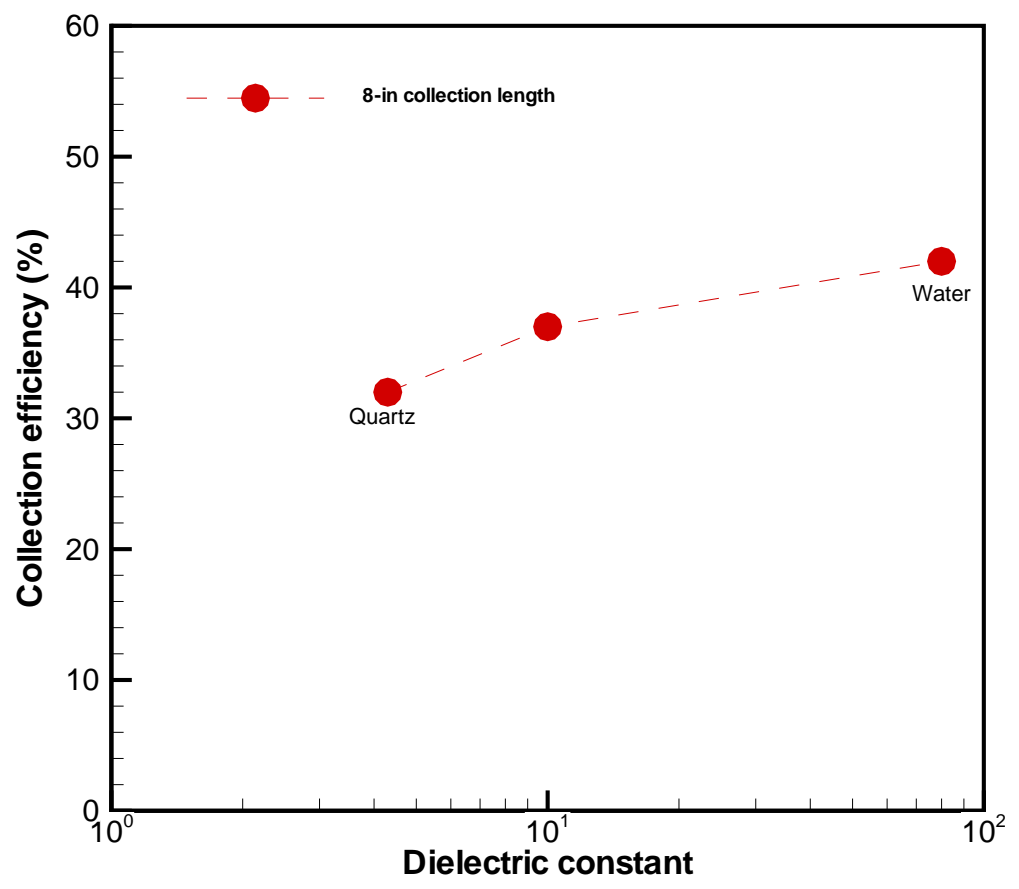

Figure 5. Collection efficiency vs. dielectric constant for 8-in collection length. 
Page: $\quad 12$ of 44

Table 3. Results of collection efficiency for various particle sizes under the reference design of collector (dielectric constant $=4.3$ (quartz))

\begin{tabular}{|c|c|c|}
\hline Particle diameter & Number of electron charges & Collection efficiency (\%) \\
\hline $0.5 \mu \mathrm{m}$ & 70 & - \\
\hline $1 \mu \mathrm{m}$ & 237 & 23 \\
\hline $2 \mu \mathrm{m}$ & 888 & 39 \\
\hline
\end{tabular}

Table 4. Results of collection efficiency for various particle sizes for the reference design collector (dielectric constant $=10$ ).

\begin{tabular}{|c|c|c|}
\hline Particle diameter & Number of electron charges & Collection efficiency (\%) \\
\hline $0.5 \mu \mathrm{m}$ & 78 & 16 \\
\hline $1 \mu \mathrm{m}$ & 276 & 26 \\
\hline $2 \mu \mathrm{m}$ & 1060 & 47 \\
\hline
\end{tabular}

Collection length The length of the collection region doesn't affect the charge on the particles, but it does affect the collection efficiency by influencing the time available for particle collection to occur. For a fixed air flow rate, a longer collection region corresponds to a longer time for the charged particle to reach the collection surface. The collection efficiency is shown in Figure 7 for two different collection lengths. 


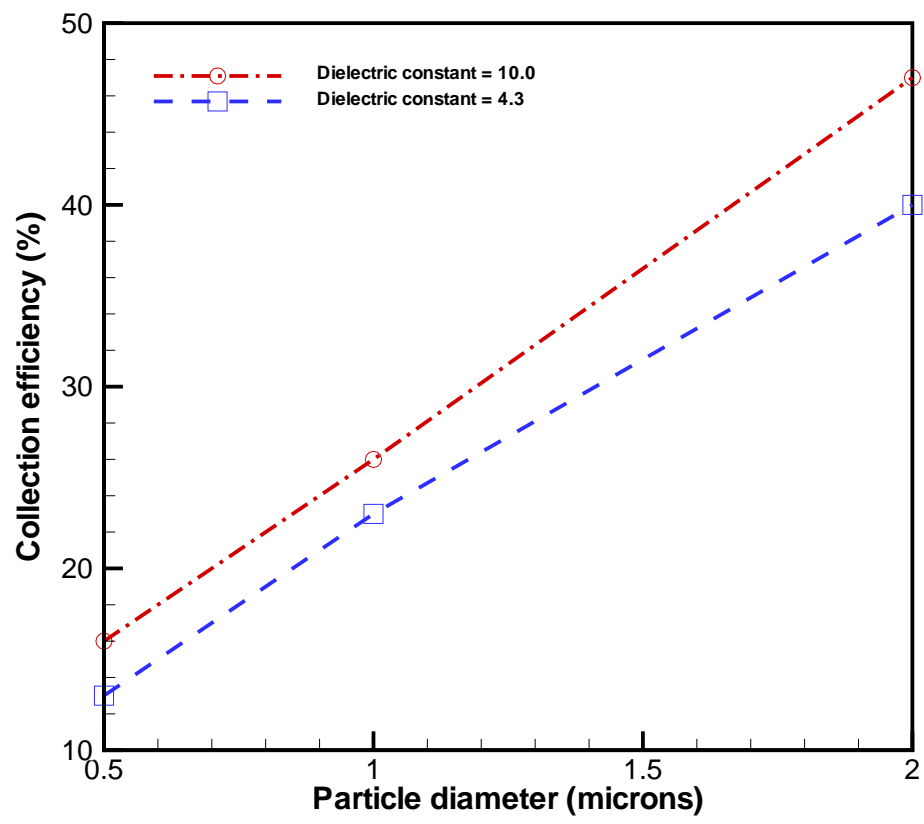

Figure 6. Collection efficiencies vs. particle size for two dielectric constants and the reference design.

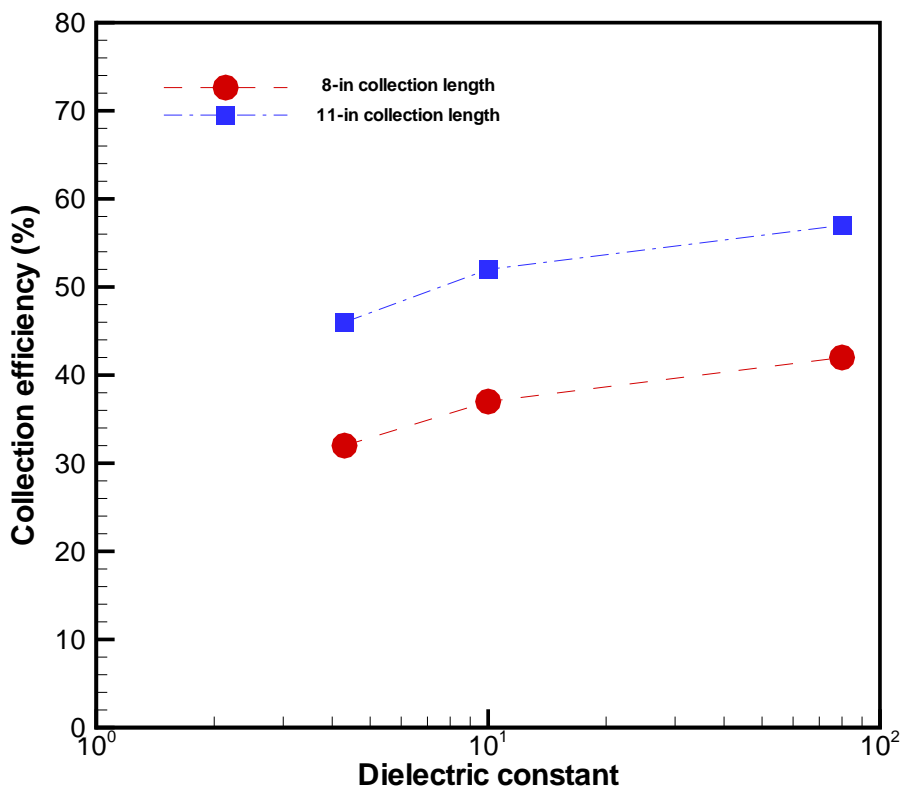

Figure 7. Collection efficiency vs. dielectric constant for two different collection lengths. 


\section{Group 2 Calculations - Modified Geometries}

Several geometric changes were evaluated in an attempt to move the incoming particles closer to the collection surface without introducing an unacceptable pressure loss in the flow channel. These changes were listed earlier and are repeated here for convenience.

The geometry variations evaluated in this analysis were:
a. Center plate
b. L-shaped inlet
c. Curved inlet
d. Smoothed outlet
e. Collection plate single obstruction
f. Collection plate multiple obstructions

Calculations for different inlet geometries were performed to test the effectiveness of forcing the particles closer to the collection surface before they entered the region of the imposed electric field. The purpose was to reduce the distance the particles had to travel to impact the surface and therefore reduce the time needed to be captured. As a rule, they did not have the desired effect of improving particle collection efficiency. One change was effective, however. That was to place a center plate in the middle of the collection region to attract the particles from both sides. The effectiveness of this geometry was a result of doubling the collection area, reducing the travel distance to the collection surface, yet maintaining the cross-sectional area for the air flow constant so the exposure time for collection remained the same as in the reference design. As expected, the collection efficiency approximately doubled for this case.

Most of the other geometric changes tried were ineffective. One reason for this was that the pressure drop through the collector was sensitive to the geometrical modifications imposed. In those cases where a smoothed inlet region was used to squeeze the flow into a smaller cross-section in the collector region (same 4-inch width but a reduced height), the increase in $\Delta \mathrm{P}$ was unacceptably large. An increased pressure drop would result in a greater power needed to drive the required $1000 \mathrm{l} / \mathrm{min}$ air flow. The contribution to the pressure drop came from both the compression region at the inlet (even though it was computationally smooth) and the collection region itself as a function of the higher air velocity. Different compression geometries were tested, but no acceptable shapes were found.

One geometric improvement was found, however, and it resulted in improved air flow performance. The original device had an L-shaped exit geometry. A recommendation was made to straighten the outlet so there were no turns in the air flow, and to close a gap between the collector region and the outlet plenum in order to prevent backflow. These changes reduced the outlet pressure drop substantially and resulted in an increased air flow for the same driving fans. Later modifications to accommodate a 2000-Ipm flow rate included smoothing the transition region between the collection plates and the outlet fans to reduce the pressure loss still further. These later changes were not evaluated computationally. 
A second type of geometric change was tested in which a bend was imposed in the inlet region in an attempt to use the inertia of the particles to move them closer to the collection plate without actually reducing the cross-section of the collection region. Both an L-shaped bend and a smoothly curved bend were modeled with somewhat similar results. While the geometry was effective in forcing the particles closer to the collection plate, it was also effective in forcing the air flow closer to the plate. The result was similar to reducing the cross-sectional area of the collection region, and the increased pressure drop was similar as well. The velocity of the air was increased, and while the distance to the collection plate was reduced, so was the residence time in the collector region. Ultimately, a slight improvement in collector efficiency was noted but it was not large enough to be considered significant.

Table 5. Results of collection efficiency for various designs (dielectric constant $=4.3$ (quartz))

\begin{tabular}{|c|c|c|}
\hline Collectors & $\begin{array}{c}\text { Pressure drop } \\
\text { across collection } \\
\text { area }\end{array}$ & $\begin{array}{c}\text { Collection } \\
\text { efficiency for 1 } \\
\mu \mathrm{m} \text { particle }\end{array}$ \\
\hline Reference design (1"x4", 8" long collection plate) & $4.8 \mathrm{~Pa}$ & $23 \%$ \\
\hline Middle collection plate (0.5"x4", 8" long) & $9.4 \mathrm{~Pa}$ & $41 \%$ \\
\hline Collection plate with 0.3" high flow obstruction & $28.6 \mathrm{~Pa}$ & $29 \%$ \\
\hline Collector with 90 flow direction change & $5.0 \mathrm{~Pa}$ & $23 \%$ \\
\hline
\end{tabular}

Center plate geometry The modification to the reference geometry by adding a center plate is shown in Figure 8. The center plate reduces the distance a particle has to travel to reach the collection plate, but it has only a minimal affect on the flow area of the collector. Therefore, the collection efficiency would be expected to approximately double. The calculated results shown in Table 5 show this to be the case. Table 5 also shows that the pressure drop in the collector approximately doubles, a result of the additional wall drag imposed on the flow by the center plate. Nonetheless, the pressure drop in the collector is still small and probably acceptable, depending on the fan characteristics.

Figure 9 shows the particle collection efficiency for the center plate geometry compared to the reference geometry for a range of electric field strengths. The same behavior noted in Table 6 persists over the entire range.

The flow profile in the collector is shown in Figure 10. The effect of the center plate can be seen in the low velocity flow region immediately next to the center plate. This is the region that introduces the additional drag and the resultant increased pressure drop. Overall, though, the flow can be seen to have essentially the same behavior as the reference design. Therefore, the principal impact of the center plate is to reduce the travel distance, and therefore the time required to attract a particle to the collection surface. 


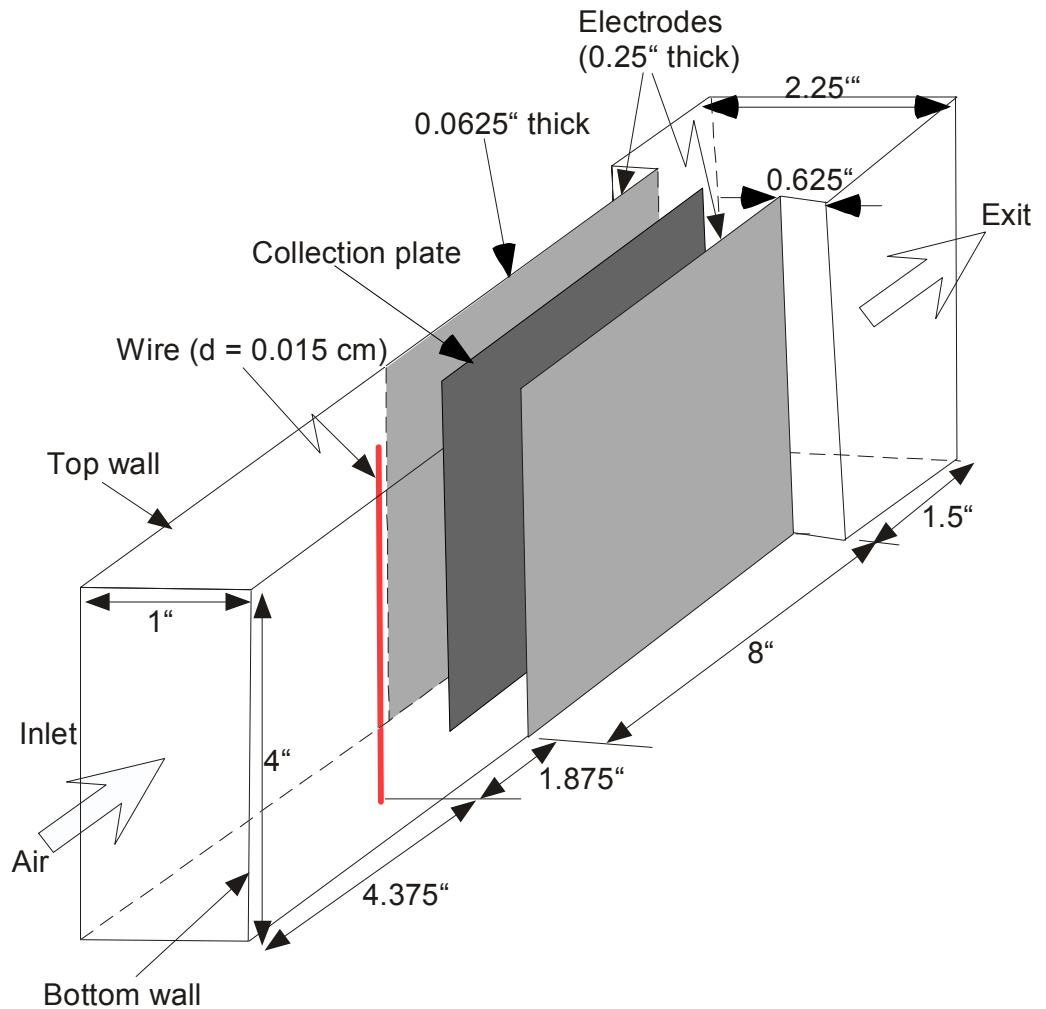

Figure 8. SRNL recommended collector geometry based on $1000 \mathrm{lpm}$ flow rate and no design restrictions. 


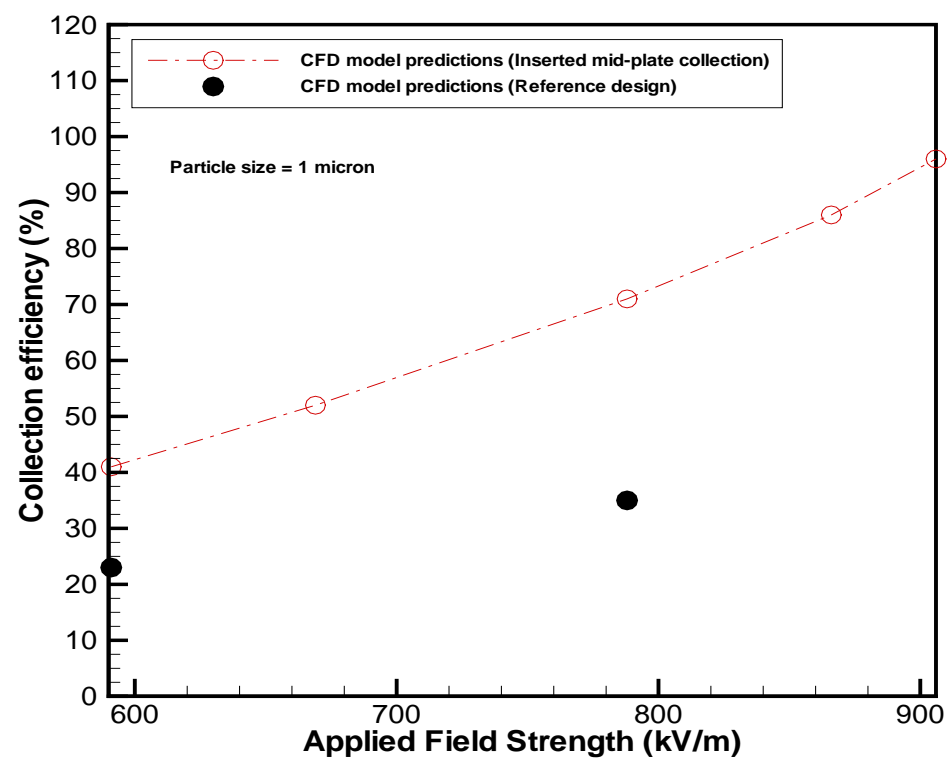

Figure 9. Comparison of collection efficiency between the two collectors for $1-\mu \mathrm{m}$ particle under 1000 liter/min

Inlet bend Two geometries were evaluated for an inlet designed to move the particles closer to the collection plate before they entered the collection region. Both implemented a $90^{\circ}$ turn in the inlet which was intended to force the entrained particles closer to the collection surface before they actually entered the collection region. Figure 11 shows the curved geometry design. The square geometry design is not shown here. The $90^{\circ}$ turn worked as expected, but it also caused the air flow itself to move toward the collection surface. The next effect was to accelerate the entire flow as if the flow area had been reduced. Therefore, while the particles moved closer to the collection surface, thereby reducing the time required to attract them to the surface, the increased velocity of the air flow tended to reduce the time available to collect those particles. The two effects tended to offset each other resulting in a minimal impact on particle collection efficiency. There was a slight increase in pressure drop, but with no measurable improvement in collection efficiency, there was little reason to explore this change further.

Figures 12 and 13 show the velocity contours and turbulence intensity profiles for the Lshaped collector. The increased turbulence intensity results from the flow expanding somewhat in the collection region after it exits the inlet region and the turn.

Curved Inlet Several modifications to the inlet shape were investigated for the purpose of slowing the inlet velocity to allow more time for the entrained particles to acquire an electrical charge in the ionization region of the collector. The modified geometry was designed to provide a greater flow area in the ionization region with a smooth transition to the collection region to minimize the pressure drop from the flow area contraction. Some representative shapes and the resulting pressure drops are shown in Table 6. The results show a significant increase in the pressure drop from the 
2:1 compression compared to the drop in the reference geometry. They also show that simply lengthening the entrance region without changing the flow area results in only a minimal change in the overall pressure drop. Figure 14 is a characteristic flow pattern. 

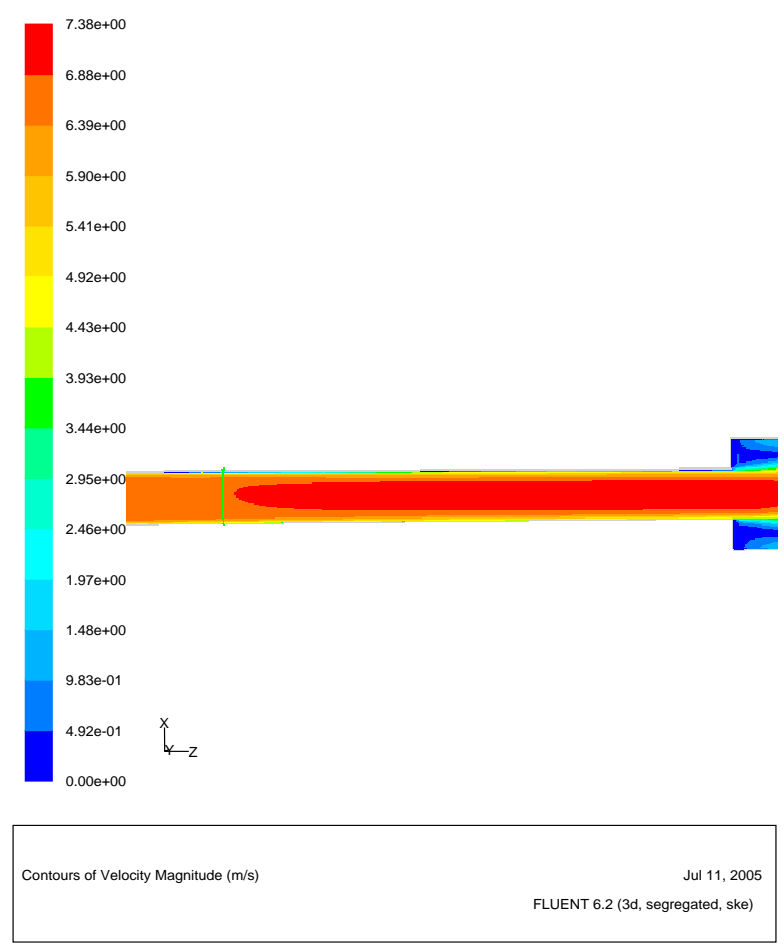

(Reference case)

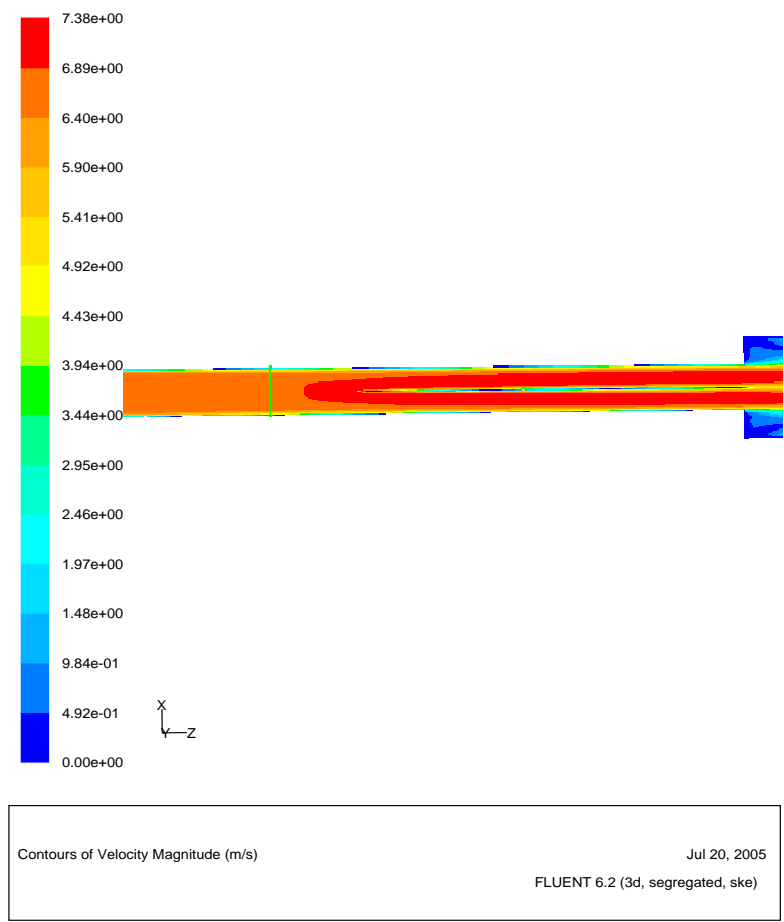

(Mid-collection plate case)

Figure 10. Comparison of velocity contours between the two collectors for $1-\mu \mathrm{m}$ particle under 1000 liter/min 


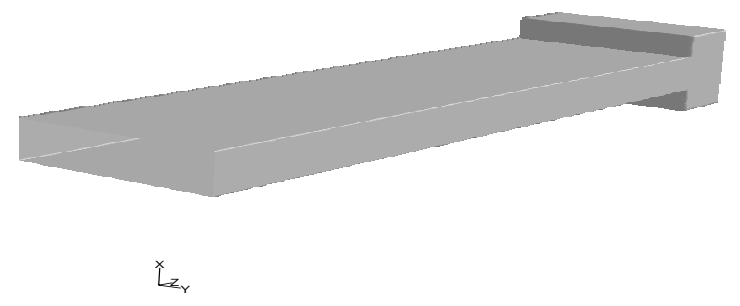

(Reference Case)

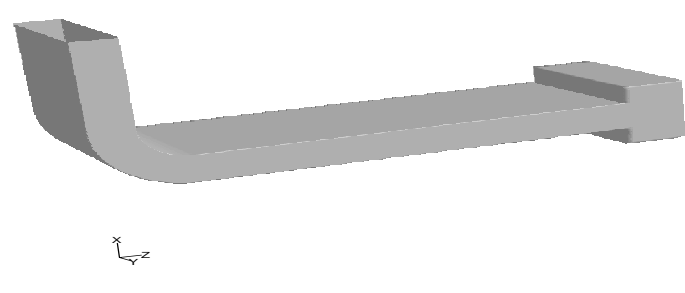

(L-type collector Case)

Figure 11. CFD models for the reference and L-type collectors 

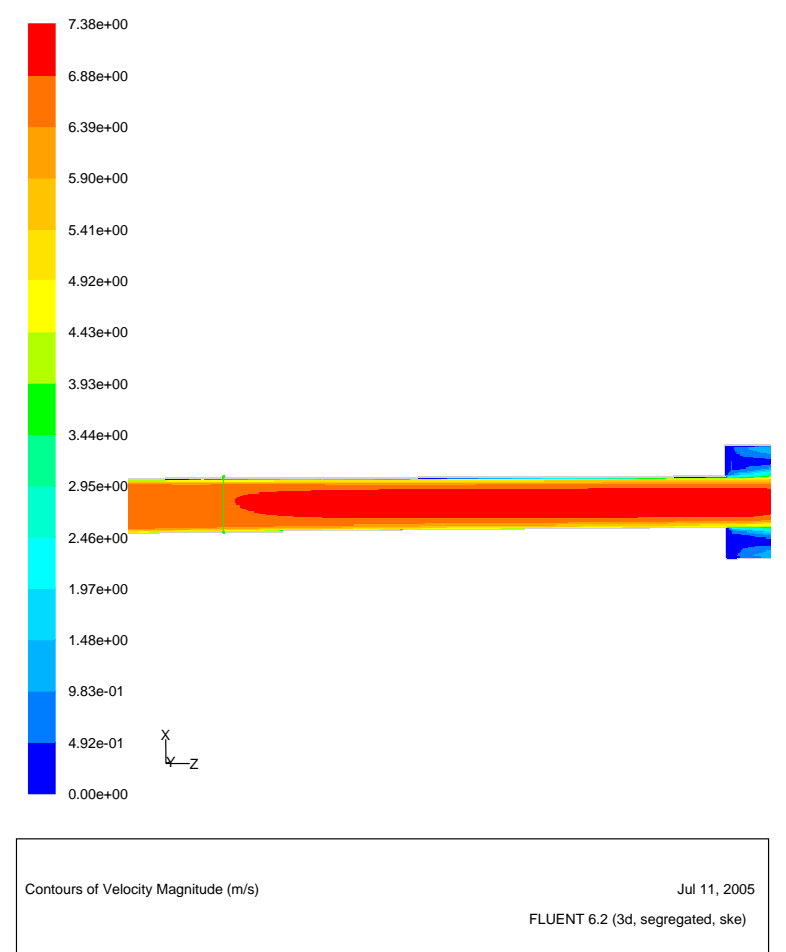

(Reference case)

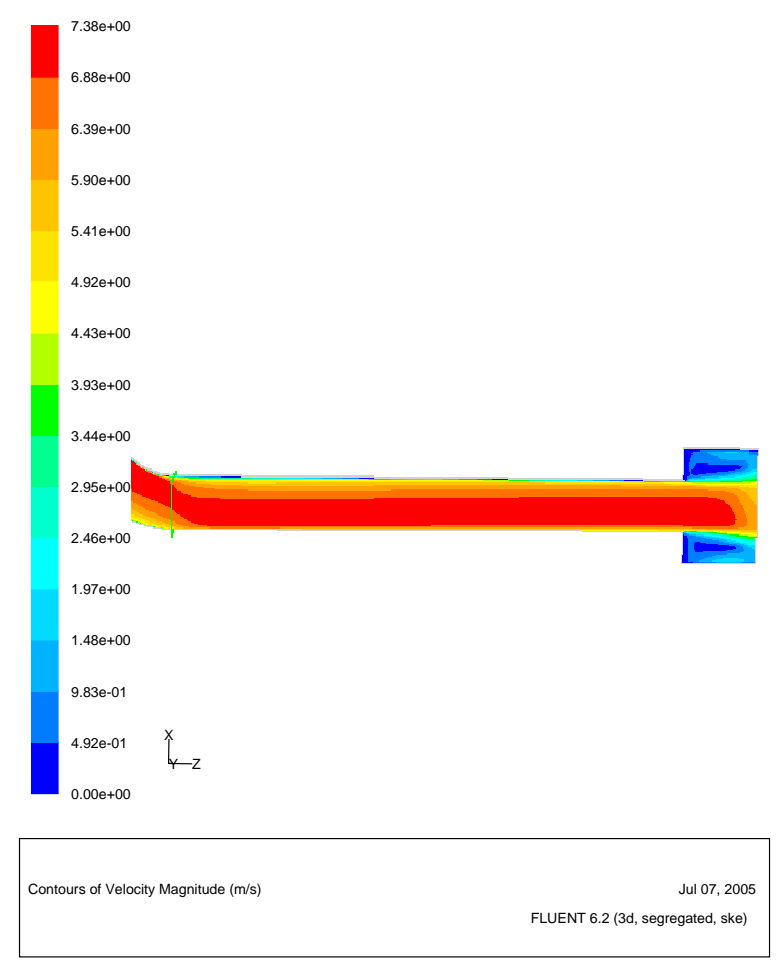

(L-type collector case)

Figure 12. Comparison of velocity contours for the reference and L-type collectors 


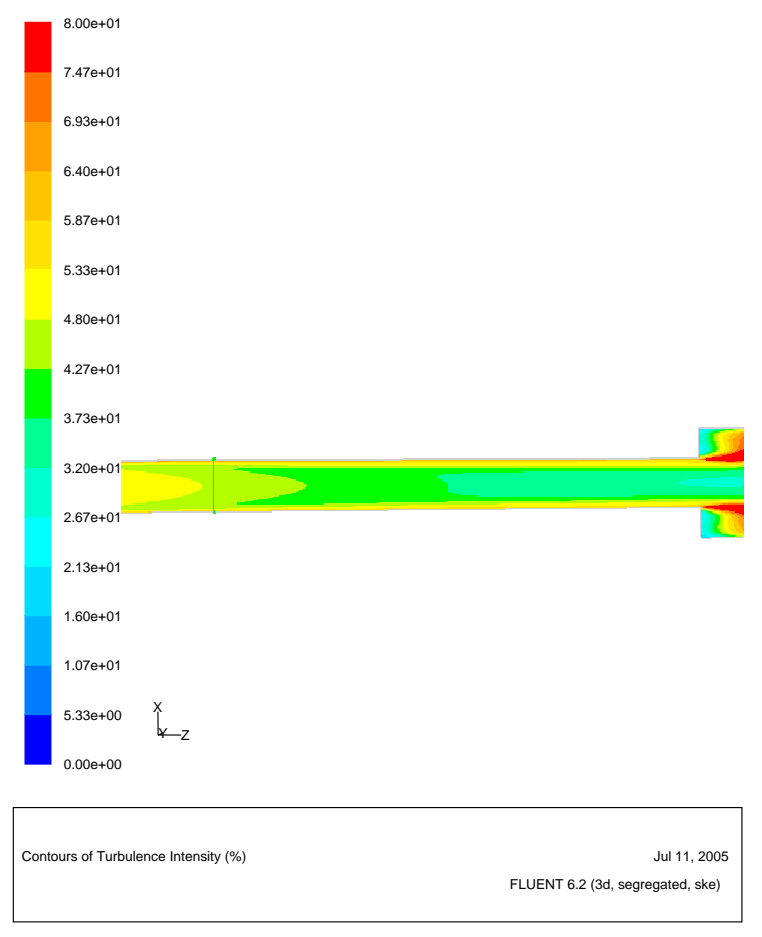

(Reference case)
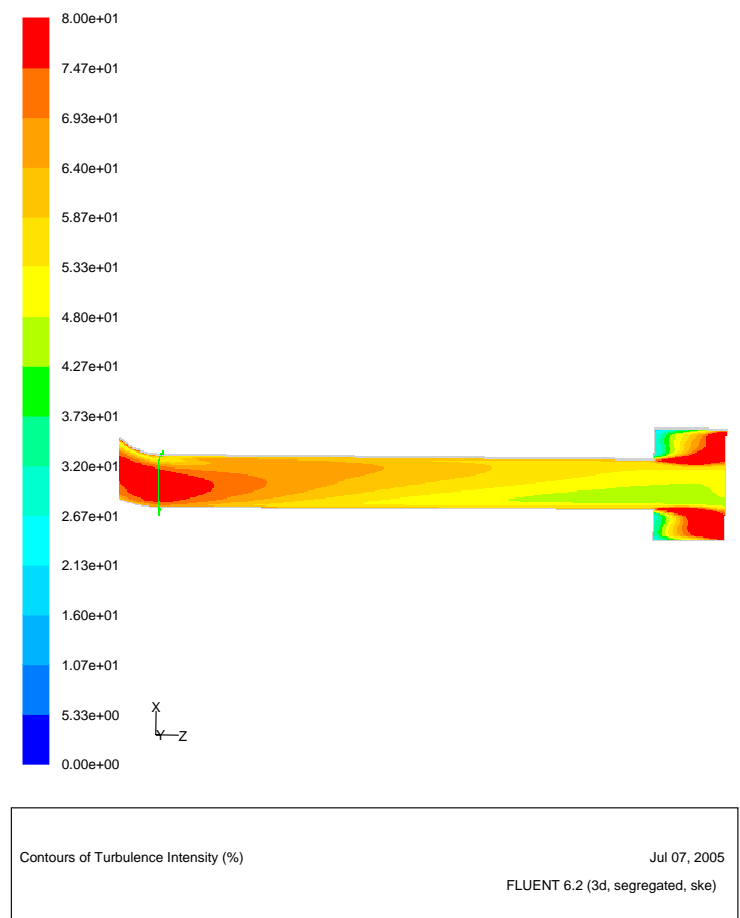

(L-type collector case)

Figure 13. Comparison of the turbulent intensities for the reference and L-type collectors 
Table 6. Power requirement comparison for different inlet geometries to the collection region
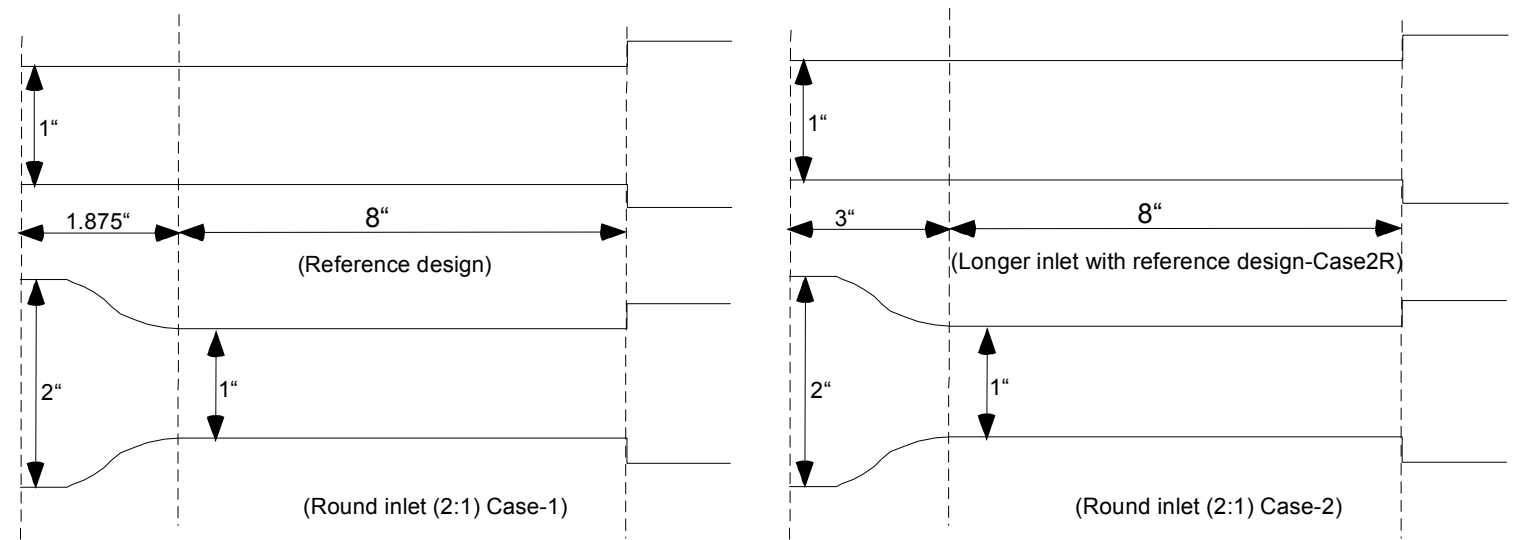

\begin{tabular}{|c|c|}
\hline Collection inlet geometry & Pressure drop through collection region \\
\hline Reference design (1:1 ratio) & $10.35 \mathrm{~Pa}$ \\
\hline Round inlet $(2: 1$ ratio -Case-1) & $30.81 \mathrm{~Pa}$ \\
\hline $\begin{array}{l}\text { Longer inlet with reference inlet } \\
\text { geometry }(1: 1 \text { ratio }- \text { Case-2R) }\end{array}$ & $12.92 \mathrm{~Pa}$ \\
\hline Round inlet $(2: 1$ ratio -Case-2) & $31.09 \mathrm{~Pa}$ \\
\hline
\end{tabular}




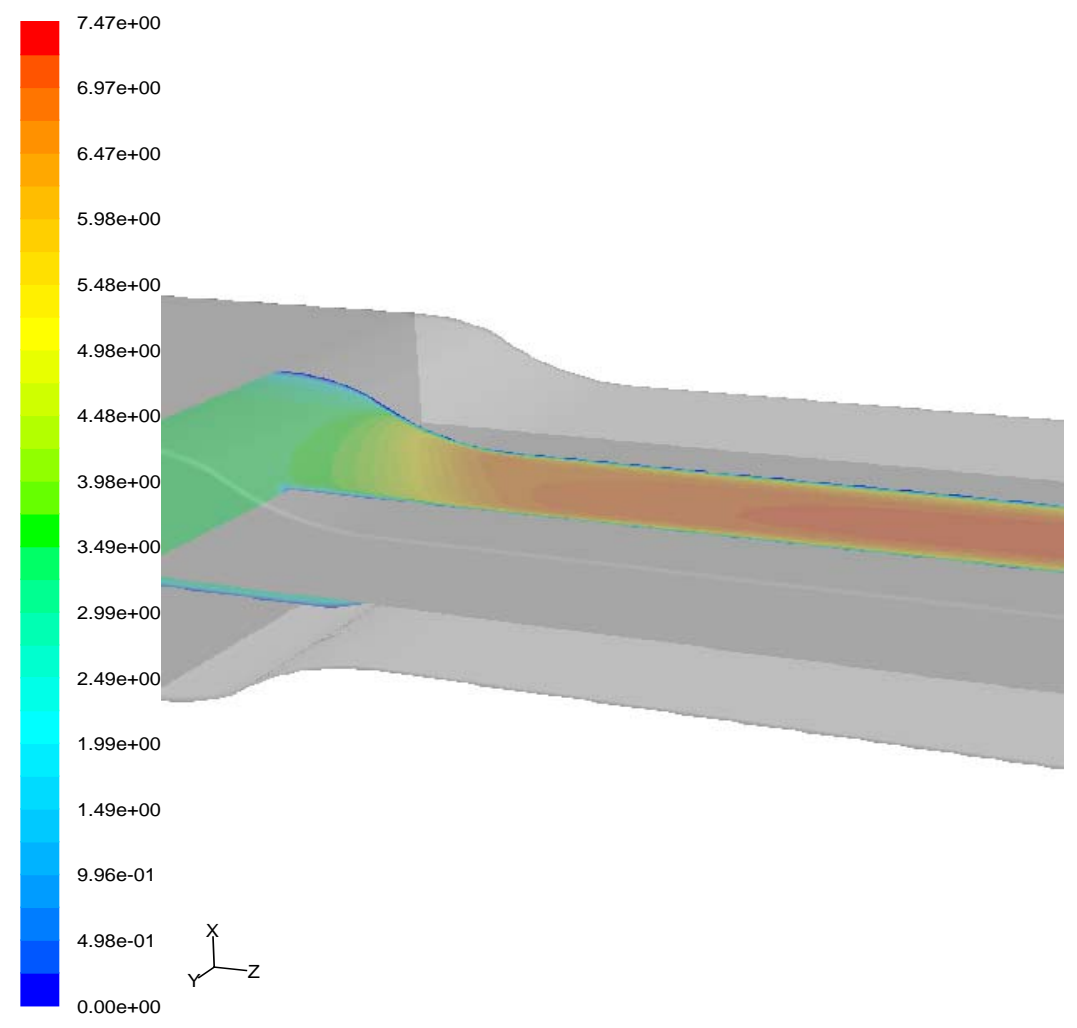

Figure 14. Velocity contour for 1.8-in separation gap distance for $2: 1$ transition region

Obstructions While the electrostatic charge on the particles provides the principal force to attract the particles to the collection surface, the mechanical effect of drag from the air flow can be utilized to assist in collecting the particles. This was shown to be effective in the case of introducing a $90^{\circ}$ bend in the entrance region, even though the impact of compressing the flow counteracted the effect of moving the particles closer to the collection surface. In this same vein, obstructions were placed in the entrance of the collection region to induce eddys that would tend to carry the entrained particles toward the collection surface and improve the collection efficiency.

Two sets of obstructions were evaluated. The first was a single obstruction 0.3 inches high on the lower surface. The second was a series of four obstructions approximately 0.1 inches high, also located on the lower surface. The flow patterns shown in Figures 15 and 16 show that the single obstruction is effective in creating an eddy region downstream of the obstruction that enhances the collection efficiency of the collector. 

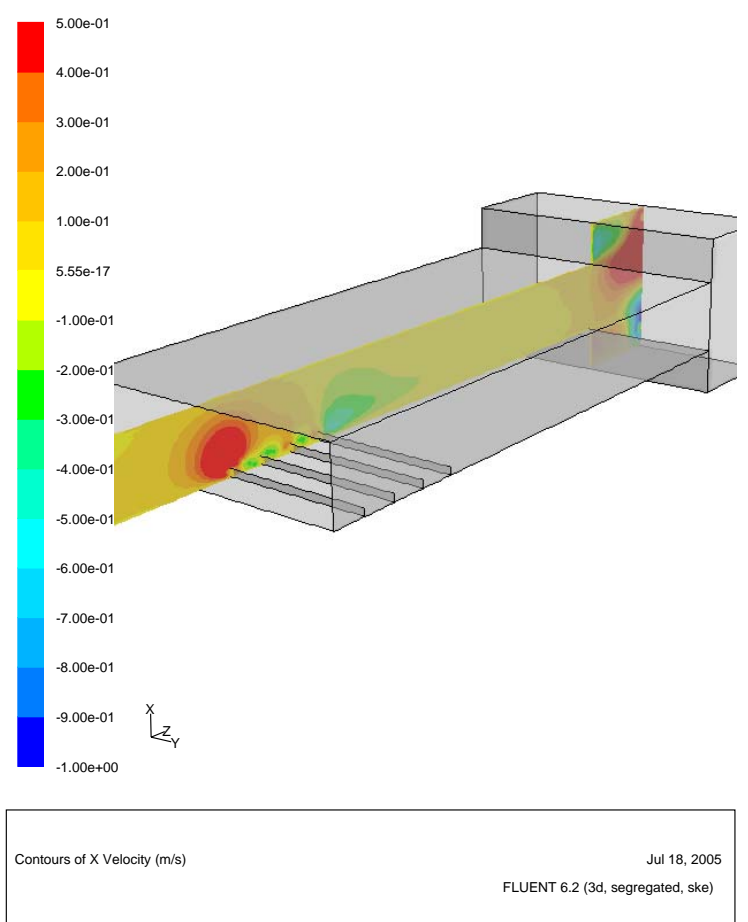

(Multiple flow obstructions on the collection surface)
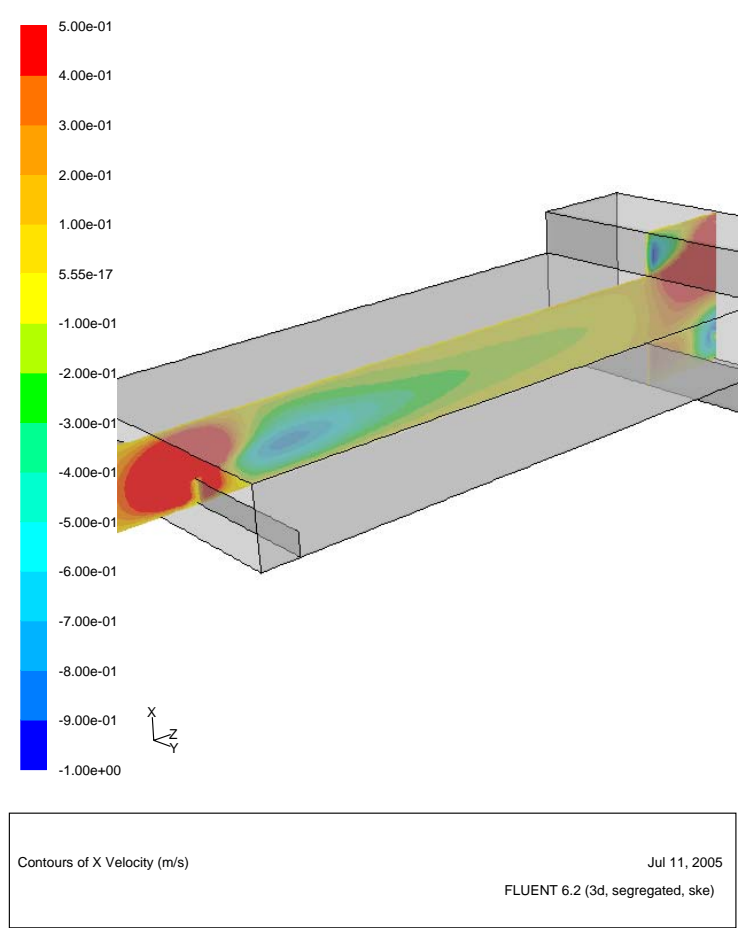

(Single flow obstruction on the collection surface)

Figure 15. Comparison of vertical velocity components for the collectors with different wall surface conditions 


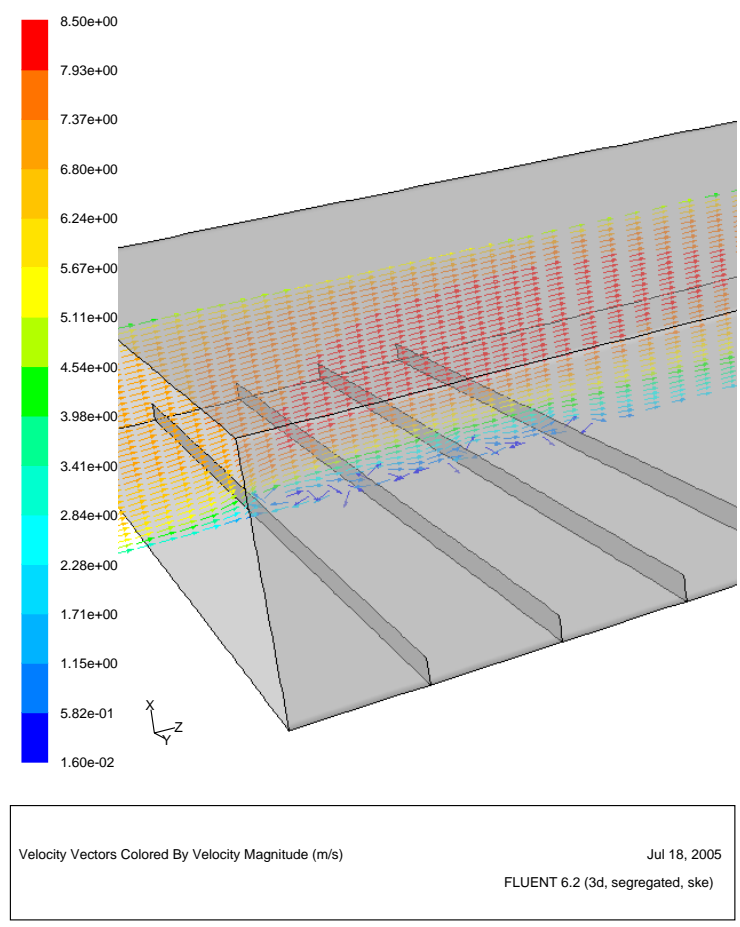

(Multiple flow obstructions on the collection surface)
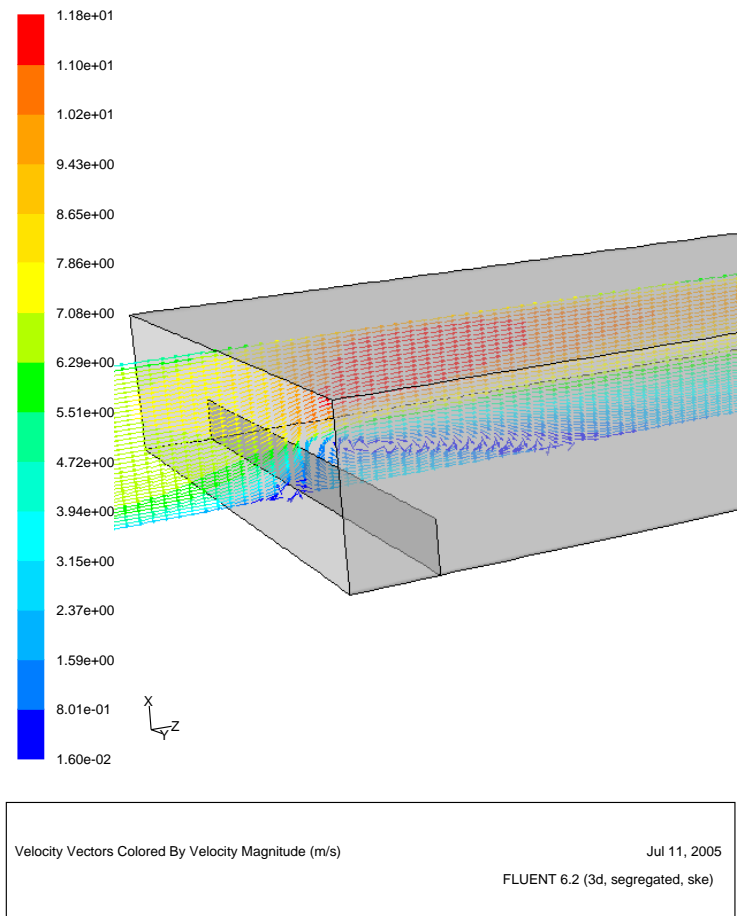

(Single flow obstruction on the collection surface)

Figure 16. Comparison of flow patterns for the collectors with different wall surface conditions 


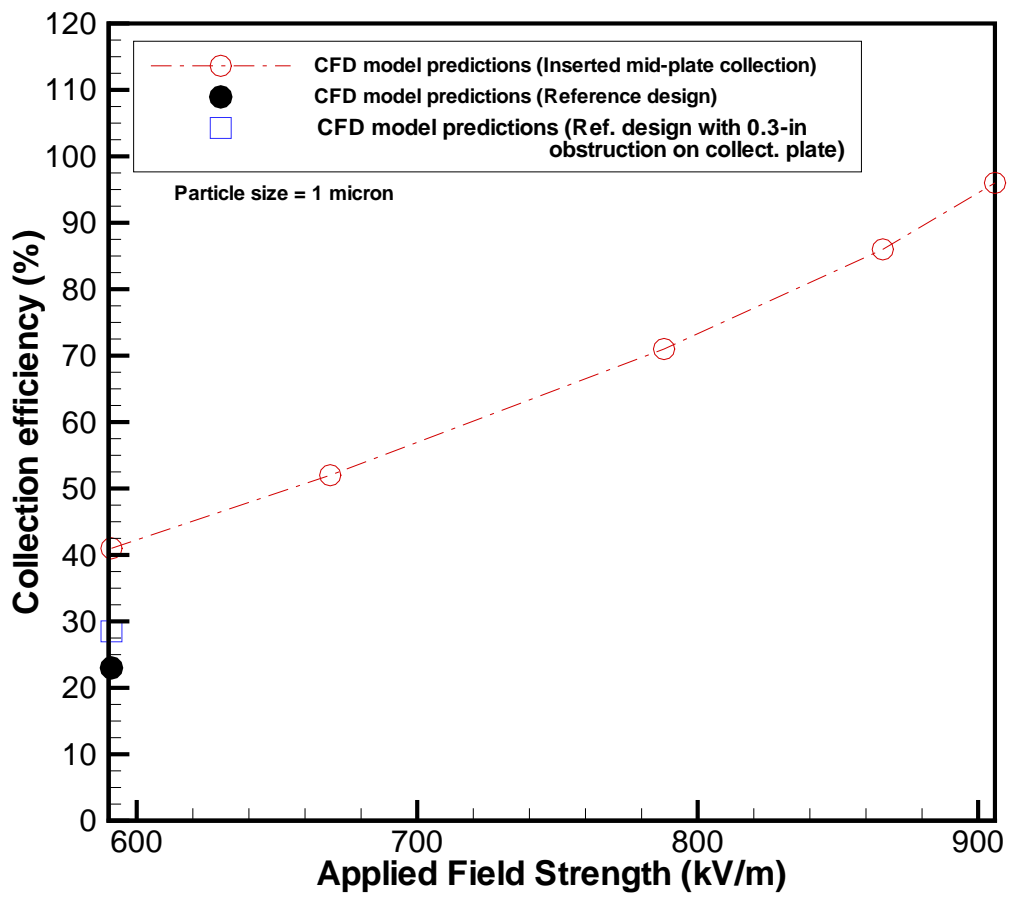

Figure 17. Collection plate with 0.3 -in flow obstruction near the entrance of collector region

The impact of the multiple obstructions is different from the single obstruction in that the flow tends to shift toward the upper plate, so rather than create a series of eddys that move particles toward the collection surface, the multiple obstructions have exactly the opposite effect and move the flow and the entrained particles away from the collection surface. Because of this effect, the multiple obstruction geometry was not investigated further.

The effect of the single obstruction on collector efficiency in shown in Figure 17 and compared to the reference design and the middle collection plate geometry. The single obstruction introduces a small improvement in collection efficiency compared to the reference design.

Figure 15 also shows the effect of the expansion at the exit of the collection region. It was noted earlier that some modifications to this region were recommended to remove an L-shaped exit and an open area between the flow region and the collector housing. Both of these recommendations were implemented with a resulting reduction in the overall pressure drop in the collector and an increase in total air flow. 


\section{Discussion}

A number of changes to the collector design were investigated in an attempt to use mechanical forces acting on the particles entrained in the air flowing through the collector to supplement the electrostatic force and improve the overall collector efficiency. With the exception of adding a collector plate in the middle of the flow region, none of the changes improved the collection efficiency enough to justify the increased pressure drop that accompanied the change. At the time that these analyses were being performed, there was no consideration given to increasing the capacity of the driving fans, so pressure drop was an important consideration. Since that time, the collector has been modified to increase the overall flow rate. The power requirements for the fans have been given less importance than collection efficiency at this stage in the development process. Nonetheless, the minor improvements in collection efficiency resulting from the geometric changes evaluated here don't warrant changes to the collector, with the possible exception of the center collection plate.

\section{Conclusions}

The charge carried by the particles is important to the ability of the collector to capture them. The analysis completed thus far has demonstrated that this effect can be captured by Fluent and that comparisons with results reported in the literature generally show good agreement.

The analyses also showed that geometric changes to the design can be important, but that the effect on the particles cannot be considered independently of the air in which they are carried. Therefore, a coupled analysis should be completed before attempting to evaluate geometrical changes physically. In general, the computational models are fairly quick to develop and run and allow the researcher to explore many different options to find one that seems promising. This can serve to keep experimental costs reasonable by focusing them on the geometries that are most likely to give significant improvements. In the work reported here, the most (and to date, only) promising modification was the addition of the center collection plate.

\section{Further Areas for Study}

Collection surface: The flow characteristics of the collection surface, in particular the roughness of the surface, are thought to impact the development and thickness of the boundary layer and the degree of turbulence intensity in the flow near the surface. The impact of this behavior on particle collection is not well understood, nor has it been addressed in this study. Nonetheless, it is thought to be important and worth exploring in future work.

Turbulence: The impact of turbulence in the free stream is qualitative in the present analysis. the intensity of the turbulence can impact the distribution of particles as they travel through the collection region, but the extent to which particle motion is affected has not been quantified. Comparisons between the Fluent results and the DeutschAnderson equation as discussed in Hinds point this out. Hinds' discussion of the Deutsch-Anderson equation describes a particle distribution that remains uniform 
throughout the entire cross-section of the collector. This results in the exponential form of the equation as particles are removed from that differential layer close to the surface and the rest are immediately and uniformly redistributed. The Fluent calculation, on the other hand, imposes a net force on the particles and attracts them to the collection surface in much the same way as would be expected in a laminar flow. This behavior would be qualitatively correct if the electrostatic force is large compared to the force imposed by the turbulent eddies in the flow, but the magnitude of the turbulent eddies and their influence on particle motion has not been evaluated. It is an appropriate area for additional work.

Humidity: The impact of humidity on collector efficiency is uncertain. The arguments supporting its influence tend to outweigh those that dismiss it, but even the supporting arguments leave questions about the specific mechanism by which particle collection is affected by humidity. Particle surface area, particle agglomeration, and electric field strength are all potentially influenced. As mentioned above, the models employed for this analysis treated the influence of humidity through the magnitude of the charge on the aerosol particles. A more mechanistic treatment of this parameter leading to a better understanding of its influence is appropriate.

\section{References}

1. Cetnar, Jerzy 2006. General solution of Bateman equations for nuclear transformations, Annals of Nuclear Energy 33, 640-645.

2. Cochet, R. 1961, "Lois Charge des Fines Particules (Submicroniques) Etudes Theoriques - Controles Recents Spectre de Particules", Coll. Int. la Physique des Forces Electrostatiques et Leurs Application, Centre National de la Recherche Scientifique, Paris, Vol. 102, pp. 331-338.

3. Hinds, W. C. 1999. Aerosol Technology, John Wiley \& Sons, Inc., New York.

4. Kim, S. H., Park, H.S., and Lee, K. W. 2001. Theoretical model of electrostatic precipitator performance for collecting polydisperse particles, J. Electrostatics 50, pp. 177-190.

5. Li, A.and Ahmadi, G. 1993. "Aerosol Particle Deposition with Electrostatic Attraction in a Turbulent Channel Flow", J. of Colloid and Interface Science, Vol. 158, pp. 476482. 


\title{
Appendix A
}

\section{Engineering Design of a Particle Collector}

\author{
Andrew M. Casella and R. A. Dimenna
}

\section{Introduction}

As air flows through a duct, particles that are suspended in the air tend to settle out and stick to the walls of the duct. The parameters that affect how many of these particles fall out of suspension are the volumetric flow rate $(Q)$ of the air, the interior surface area $\left(A_{c}\right)$ of the duct, and the settling velocity $\left(V_{s}\right)$ of the suspended particles. The extent to which these parameters influence particle deposition depends on whether the air flow is laminar or turbulent.

The case of laminar flow in a horizontal, rectangular duct is straightforward. The trajectory of a suspended particle will be a smooth line that is determined by its velocity in the axial (horizontal) direction due to the flow of the gas and its velocity in the perpendicular (vertical) direction due to its settling velocity. Since the flow is laminar, the change in each of these velocities at any given time is predictable. Thus, the particle trajectories are smooth. The number of particles that settle out of suspension at any incremental time is equal to the number of particles that are located close enough to the duct wall that their settling velocity will enable them to come into contact with the wall during the time increment. This relationship is given by Equation $A-1$ where $N(t)$ is the number of particles in suspension at time $t, N_{0}$ is the initial number of particles in suspension, and $\mathrm{W}$ is the width of the duct. Notice that Equation A-1 assumes that the particles are uniformly distributed in the air and it describes a plane perpendicular to the $\mathrm{x}$-direction. $\mathrm{WV}_{\mathrm{s}} \mathrm{dt}$ is the area from which all particles are deposited due to their settling velocity and $\mathrm{WH}$ is the area of the entire plane.

$$
d N(t)=\frac{-N_{0} W V_{s} d t}{W H}=\frac{-N_{0} V_{s} d t}{H}
$$

Equation A-1

The amount of time that a given particle will be located inside the duct is given by the quotient of the duct volume and the volumetric flow rate of the air. This relationship is given by Equation A-2. As time progresses, the plane that we are concerned with progresses forward in the $x$ direction while all other parameters stay the same. Thus, Equation $A-3$ is the differential form of Equation A-2. Substituting Equation A-3 into Equation $A-1$ and integrating leads to Equation $A-4$. If Equation $A-4$ is integrated from $x$ $=0$ to $x=L$, and it is noticed that $L \times W$ is simply the collection area $\left(A_{c}\right)$, then Equation A-5 results. The efficiency with which the particles are deposited in the duct is given by Equation 6. Substituting Equation A-5 into Equation A-6 gives us Equation A-7 which is a description of the efficiency of particle collection for laminar flow in a rectangular duct.

$$
t=\frac{W H x}{Q}
$$

Equation A-2 


$$
\begin{aligned}
& d t=\frac{W H d x}{Q} \\
& \int_{N_{0}}^{N(x)} d N(x)=\int_{0}^{x} \frac{-N_{0} V_{s}}{H} \frac{W H d x}{Q}=\int_{0}^{x} \frac{-N_{0} V_{s} W d x}{Q} \\
& N(L)-N_{0}=\frac{-N_{0} V_{s} A_{c}}{Q} \\
& \text { Eff }=\frac{N_{0}-N(L)}{N_{0}} \\
& \text { Eff }=\frac{V_{s} A_{c}}{Q}
\end{aligned}
$$

Equation A-5

Equation A-6

Equation A-7

Notice that Equation A-7 shows that the collection efficiency for laminar flow in a duct is linearly related to the flow rate, the settling velocity, and the collection area. Thus, it is theoretically possible to design a collector that will collect $100 \%$ of the suspended particles. Take note that Equation A-7 is only valid for $N(L) \leq N_{0}$. This makes sense for laminar flow. Now consider turbulent flow in a rectangular duct.

What makes the turbulent situation mathematically different than the laminar situation is the fact that for each particle, the flow velocity in the $x$-direction and the settling velocity in the z- direction both vary with time. Thus, for our plane of interest, the number of particles $\mathrm{N}(\mathrm{t})$ can increase or decrease as particles flow through it in addition to the loss due to deposition. We still assume however, that any particle that makes it into the area $\mathrm{WV}_{\mathrm{s}} \mathrm{dt}$ will be deposited and that the particles are uniformly distributed. This implies that the number of particles lost in any infinitesimal time increment will be equal once again to the total number of particles in the plane times the ratio of the area from which all particles are deposited to the total area of the plane. This relationship is presented by Equation A-8. Substituting Equation A-3 into Equation A-8, rearranging, integrating from $x=0$ to $x=L$, and using $W x L=A_{c}$, leads to Equation $A-9$. Rearranging Equation $A-9$ and substituting it into Equation A-6 leads to Equation $A-10$, which is known as the Deutsch-Anderson equation. ${ }^{1(p p . ~}{ }^{339)}$ Equation $A-10$ is also simply known as the Deutsch equation or the Deutsch model. ${ }^{2(p p .6)}$ Notice that Equation A-10 involves an exponential that implies that $100 \%$ collection efficiency can never be reached.

$$
\begin{aligned}
& d N(t)=-N(t) \frac{W V_{s} d t}{W H}=-N(t) \frac{V_{s} d t}{H} \\
& \ln \left(\frac{N(L)}{N_{0}}\right)=\frac{-V_{s} A_{c}}{Q} \\
& \eta_{D A}=1-\exp \left(-\frac{V_{s} A_{c}}{Q}\right)
\end{aligned}
$$

Equation A-8

Equation A-9

Equation $\mathrm{A}-10$ 


\section{Results of Literature Review:}

Equation A-7 describes a purely laminar flow and Equation A-10 describes a purely turbulent flow. Purely laminar flow means that there is no turbulent mixing, while purely turbulent flow is treated as perfect mixing. Many have claimed that in reality, neither of these two cases actually exists. Instead, an intermediate situation describes the flow of an aerosol through a duct. In such an intermediate case, finite mixing is said to occur. In addition, if the aerosol particles are charged and an electric field is applied in the collector, then the electrostatic force is dominant in the collector. In this case, the collector is called an electrostatic precipitator and the settling velocity is replaced with the terminal electrostatic velocity $V_{\mathrm{TE}}$. Several finite mixing models have been created in an attempt to accurately describe the collection efficiency of an electrostatic precipitator.

The first adjustment to the Deutsch model was by Cooperman in $1971 .^{3}$ Successive improvements were made by Leonard et. al. ${ }^{4}$ in 1982 and Zhibin et. al. ${ }^{5}$ in 1992 . These models are displayed below as Equation A-11 through Equation A-13:

$$
\begin{aligned}
& \eta_{\text {Coo }}=1-\exp \left[\frac{U L}{2 D}-\sqrt{\left\{\left(\frac{U L}{2 D}\right)^{2}+(1-R) P e\left(\frac{L}{W}\right)^{2}\right\}}\right] \\
& \eta_{\text {Leo }}=1-\int_{0}^{1} P\left(\frac{\xi-D e}{\sqrt{2 D e / P e}}\right) d \xi \\
& \eta_{\text {Zhi }}=1-\sqrt{\frac{P e}{4 \pi D e}} \int_{0}^{1} \exp \left[-\frac{P e}{4 D e}(\xi-D e)^{2}\right] d \xi
\end{aligned}
$$

Equation A-11

Equation $\mathrm{A}-12$

Equation A-13

where $V_{t}$ is the migration velocity $\left(q_{p} E C_{d} / 3 \pi \mu d_{p}\right), C_{c}$ is the slip correction factor

$\left(1+\left(2 / P d_{p}\right)\left[6.32+2.01 \exp \left(-0.1095 P_{p}\right)\right]\right), P$ is the absolute pressure $(6 \mathrm{~cm} \mathrm{Hg}), E$ is the electric field intensity $\left(\mathrm{V}_{\mathrm{w}} / \mathrm{W}\right), \mathrm{W}$ is the width of wire-to-plate, De is the Deutsch number $\left(V_{t} L / U W\right), P e$ is the electric Peclet number $\left(V_{t} W / D_{p}\right), D_{p}$ is the particle diffusivity, and $P(z)$ in Equation A-12 is the Gaussian probability distribution function given by Equation A$14 .^{2}$

$$
P(z)=\frac{1}{\sqrt{2 \pi}} \int_{-\infty}^{z} \exp \left(-\frac{B^{2}}{2}\right) d B
$$

Equation $\mathrm{A}-14$

The models get increasingly more accurate, but also increasingly more complicated and difficult to evaluate for a given system. In their paper, Zhibin et al. claim that their model is the most accurate, especially for low flow rates. This is true, but Park and Kim claim that the Zhibin model is the most accurate of the four for high as well as low flow rates. In fact, they claim that the Zhibin model remains accurate as several system parameters are varied. ${ }^{2}$ Xiangrong et al. tried to improve upon the models listed above by introducing new boundary conditions that they deemed more appropriate and claimed yielded results that more closely followed experimental data. ${ }^{6}$ The boundary condition that they suggest is a zero flux condition for the turbulent mixing diffusion model at the wire plane of the wire-plate ESP. Park and Kim introduced the idea of taking into consideration the effect of particle space charges on particle behavior. ${ }^{7,8}$ They noticed that charged particles influence the electric field significantly and that the electric field in the collector portion of the ESP is not uniform in reality. Varying values of the particle space charge resulted in noticeably different particle behavior in computational models. 
Thus, they concluded that keeping space charge in models will help in model accuracy and in developing more efficient ESPs. The effect of space charge on particle behavior was also studied by Choi and Fletcher, but for a one stage ESP. ${ }^{9}$ They developed a FLUENT model that took into consideration particle charges. They concluded that, "...significant decrease in corona current and the distorted distribution of ion charge due to the particle space charge are observed for small particles with relatively high dust loading by the calculation of strongly coupled governing equations. The effect of particle space charge on the particle transport is also considerable."

Whatever model is used, the fact remains that there are several physical factors that influence the collection efficiency of the ESP. The successful engineering of an ESP depends ultimately on how efficiently particles can be made to contact the collector surface and how efficiently they can be made to stay there. Large collector areas and low volumetric flow rates help to increase efficiency because they increase the amount of time that the collector has to capture particles. If the maximum allowable collector size and minimum allowable flow rate have been reached and the collector efficiency is not as high as desired, then stronger forces must be applied to get the particles to the collection wall quicker. The way that this is done in an ESP is to ensure that particles to be collected acquire a maximum number of fundamental charges and that the strongest possible electric collection field is applied.

Particles acquire charges through two mechanisms: field charging and diffusion charging. In field charging, electrons or ions follow electric field lines. The electrons or ions collide with and stick to the particle which thus acquires charge. The probability that a particle will be struck by an electron or ion is proportional to the surface area of the particle and thus the square of its diameter. Equation A-15 describes the number of elementary charges that particles of a given diameter should acquire when subjected to an electric field as a function of time. Since field charging is a function of the square of the particle diameter, it affects larger particles to a much greater extent. In diffusion charging, ions and particles collide as a result of mechanical motion. When these collisions take place, charges are transferred between the colliding entities. This mechanism is a function of particle diameter. The number of elementary charges that a particle of a given size can be expected to acquire as a function of time is described by Equation A-16. The charging of smaller particles is heavily governed by diffusion charging. ${ }^{1(\mathrm{pp} .325)}$

$$
\begin{aligned}
& n(t)=\left(\frac{3 \varepsilon}{\varepsilon+2}\right)\left(\frac{E d_{p}^{2}}{4 K_{E} e}\right)\left(\frac{\pi K_{E} e Z_{i} N_{i} t}{1+\pi K_{E} e Z_{i} N_{i} t}\right) \\
& n(t)=\frac{d_{p} k T}{2 K_{E} e^{2}} \ln \left(1+\frac{\pi K_{E} d_{p} \bar{c}_{i} e^{2} N_{i} t}{2 k T}\right)
\end{aligned}
$$

Equation A-15

Equation A-16

For any given particle size, the number of charges acquired is a function of Equation A15 and Equation A-16. For particles with diameters greater than $1 \mu \mathrm{m}$, Equation $\mathrm{A}-15$ gives a good estimate of the number of charges to be acquired. For particles with diameters smaller than $0.1 \mu \mathrm{m}$, Equation A-16 gives a good estimate of the number of charges to be acquired. Several models have been developed to describe the charging of particles with diameters between 0.1 and $1 \mu \mathrm{m}$. Like the models for the overall collector efficiency, the more accurate models for particle charging are more complicated and difficult to use. The most recent model is a numerical model proposed by Lawless. ${ }^{10}$ 
In general, as a rule of thumb for engineering purposes, the number of charges acquired by particles of all sizes by the ESP can be predicted to be the simple sum of the charges acquired though Equation A-15 and Equation A-16. The larger the particle, the more charges it will acquire, and thus the larger the force will be that pulls the particle to the collector plate. Smaller particles will receive fewer charges, but they also experience less drag as they move through the air and thus have a higher mobility than larger particles. For particles with diameters between 0.1 and $1 \mu \mathrm{m}$, the number of charges is small enough and the drag is large enough, that a minimum in collection will occur. ${ }^{1(p p .}$ 327) For this reason, much effort is expended on describing the behavior of particles in this size range. Collection of particles in this size range will be the limiting factor in the efficiency of the particle collector.

It is this size range of particles that plagues environmental scientists as well. Water droplets in the form of rain or clouds collide with and remove particles from the air in a process known as scavenging. The particle size range of 0.1 to $1 \mu \mathrm{m}$ is least likely to be removed from suspension in the air and is known as the "Greenfield Gap." Interestingly enough, if the Greenfield Gap particles carry a charge, then the probability of contact between a water droplet and a particle is increased by as much as an order of magnitude. Thus at the base of clouds, where charges concentrate and droplets are plentiful, the scavenging of Greenfield Gap particles is enhanced. ${ }^{11}$ This enhancement of scavenging by way of electrical charges is known as electroscavenging. Electroscavenging has already been used by Suh et al. to apply larger numbers of elementary charges to nanoparticles for use in nanoparticle pattern structures. ${ }^{12}$ The way this process works is that solvent vapor is first condensed on the particles in a device known as a particle magnifier. The droplets containing the particles are then charged with a corona wire. The number of charges acquired by the droplet is much greater than the number of charges that the particle would have acquired on its own due to the shear increase in size. After charging, the solvent is evaporated from the particles and charges are left behind. Results show that the number of charges acquired is approximately proportional to the particle diameter raised to the power of 1.9. Clark et al. also have developed a system through which particles are "grown" with humidity to larger sizes so that they can be more easily manipulated with electrical charges and fields. ${ }^{13}$ They simply introduced a supersaturated nitrogen stream to their aerosol stream prior to its entry into the corona charger and allowed the water vapor to condense on the particles effectively "growing" them to larger sizes. They claim that the factors governing the growth of particles through vapor condensation were addressed in a 1988 paper by Barrett and Clement. ${ }^{14}$

Much attention has been given to the idea that particle charges can be maximized by increasing the functioning of the corona wire charger. Essentially, corona wires work by creating a cascade of ions that are directed through a dispersion of particles in the hope that some of them will become attached to the particles. If the corona wire is given a negative polarity, then the cascade will be electrons, but if the corona wire is given a positive polarity, the cascade will be positive ions. The corona wire does not directly "emit" electrons or ions. Instead, ions that already exist in the air as a result of natural ionizing radiation are accelerated toward or away from the corona wire depending on their polarity. If these ions are accelerated to a high enough velocity, then a collision between the ion and a neutral air molecule will knock an electron loose from the neutral molecule. That electron and the positive ion that is left behind when it leaves the neutral molecule are then accelerated by the corona wire causing more collisions and electrons to be stripped from other neutral molecules. This process continues, creating an 
electron cascade in one direction and an ion cascade in the other. ${ }^{15}$ The potential at which ions are accelerated by the corona wire to a great enough velocity to strip electrons from the air is known as the breakdown potential for air. This breakdown potential is a function of the corona wire diameter and is given by Equation A-17. ${ }^{1(p p . ~ 332)}$

$$
E_{b}=3000+127 d_{w}^{-1 / 2}
$$

Equation A-17

When attempting to optimize the charging section of an ESP, the goal is essentially to maximize the ion concentration. There are several parameters that can be varied in order to achieve this. Several studies involving the variation of these parameters have been undertaken in order to improve collection efficiency. The study by Kim and Lee is exceptionally extensive. ${ }^{2}$ This study examined the wire-to-plate spacing, the wire radius, the air velocity, the turbulence intensity, the applied voltage, the wire-to-wire spacing for multiwire ESP, and the influence of particle contamination. The study concluded that faster air velocities result in lower collection efficiencies. This is just a confirmation of something that all theories since Deutsch readily predict. The study concluded that smaller corona wire diameters and smaller wire-to-plate spacing improved the collection efficiency. However, in the case of the multiwire ESP, there is an optimum wire-to-wire spacing. Having too many corona wires in a limited area causes them to be too close which degrades the collector efficiency. It reported that higher turbulence intensities result in lower efficiencies, but that this effect could be offset by increasing the collector potential to a very high value. The study concluded that the model of Zhibin and Guoquan was the best for predicting collector performance. Kim and Lee noticed that the collection efficiency decreased with time. They attributed this phenomenon to the deposition of particles on the corona wires and on the collection plate. This led to a phenomenon known as back corona.

Back corona was extensively addressed in 1979 by Thanh. ${ }^{16,17}$ He claimed that as particles flow through the corona charger, they are deposited on the corona electrode and on the collector electrode. A voltage begins to build up across these deposited particle layers until a current begins to flow through the particle layer. This causes a large potential build up to occur in the air gaps within the layer. When the potential across the air gaps reaches a sufficient value, breakdown of the air occurs. This breakdown can cause a corona to emanate from the collection electrode and possibly can cause a complete sparkover between electrodes. The back corona adversely affects the charging efficiency of the corona wire charger.

Kim and Lee suggested that back corona can be alleviated through a process known as rapping which is the mechanical removal of particles from the charger electrodes. ${ }^{2}$ The two devices suggested for rapping were hammers and vibrators. The hammers simply pound on the electrodes which ejects the particles from the electrodes. Vibrators, as one would suppose, send vibrations through the electrodes which also eject particles from the electrodes. Care must be taken in the design of the rapping system. The goal is to provide enough mechanical energy to eject the particles from the corona charging area, but not so much mechanical energy that the particles are thrown out of the detector. Ideally they will be ejected and then recaptured after moving a short distance downstream of the corona charger.

Other researchers have also seen the necessity to combat back corona. Chen and Pui developed a charger that used sheath flow for such a purpose. ${ }^{18}$ High velocity air streams were made to flow along the collector plates to prevent the particles from depositing in the charger. They used a $\mathrm{Po}^{210}$ source as an ion source so there was no 
corona wire for particles to deposit on. Kruis and Fissan developed what they called a twin Hewitt charger. ${ }^{19}$ In this device, the air is made to flow through a duct that has sides made of wire mesh. The sides are charged to opposite polarities that are flipped at an optimal frequency. This enhances diffusion charging for small particles and prevents the charged particles from depositing on the wire meshes. There is a corona wire on either side that produces an ion stream that is directed into the charging zone to increase charging efficiency. This design is much more drastic but claims to improve charging for very small particles $(\sim 0.01 \mu \mathrm{m})$ and prevents deposition of particles in the charger. Biskos et al. developed a Hewitt-type charger that employed an alternating current and sheath flow for emphasis on the diffusion charging of nanoparticles. ${ }^{20}$ The diffusion charging of small particles that the above authors were trying to achieve is directly dependent on the ion concentration created in the charger and the residence time of the particles that they are trying to charge. Hernandez-Sierra et al. measured the ion concentration for positive and negative coronas at varying voltages. ${ }^{21}$ They report ion concentrations of about $10^{6}$ per $\mathrm{cm}^{3}$ for a negative corona with a voltage of $3 \mathrm{kV}$. Their charger had much smaller dimensions and operating parameters than ours. They state that higher ion concentrations can be obtained with positive coronas, but that the positive coronas require higher voltages for operation. They also claim that more of the ions produced by the negative corona than the positive corona end up being lost through electrostatic forces to the ground plates in the charger. They also report higher losses of ions due to electrostatic deposition in the charger for higher corona voltages.

A solution that can be applied to nearly any design was presented by Sekar in $1981 .^{22}$ He noted that by increasing the relative humidity in the charger, the effects of back corona can be nearly eliminated. He proposed that the mechanism through which this is achieved is that the humidity decreases the electrical resisitivity in the air and in the deposited particle layer. Instead of building up potential in the air gaps in the deposited layer, the ion current just flows through it because the resistance is low enough. This prevents the formation of a corona backdischarge. Sekar noticed a significant reduction in the occurrence of back corona for the same conditions if the relative humidity was raised from $10 \%$ to $24 \%$. The observation that humidity greatly reduces (if not eliminates) the effects of back corona was also made by Chang and Bai. ${ }^{23}$ They noticed that for a variety of corona applied voltages, back corona would occur causing the ESP efficiency to decline with time. This effect could be eliminated by "conditioning" the air flow with a water mist. Chang and Bai concluded that, "Flue gas conditioning of water injection can effectively improve the adverse effect caused by back corona. It allows the ESP to operate under a higher effective corona power and thus a higher collection efficiency can be obtained." Sekar's claims are also supported by the EPA-CICA Air Pollution Control Technology Fact Sheet for the Wire-Plate Type Wet Electrostatic Precipitator. ${ }^{24}$ This fact sheet claims that... "Because of the high humidity in a wet ESP, the resistivity of particles is lowered, eliminating the "back corona" condition." In addition, the fact sheet claims that the wet ESP is... "capable of very high efficiencies, even for very small particles... and...The continuous or intermittent washing with a liquid eliminates the reentrainment of particles due to rapping which dry ESPs are subject to."

The fact sheet mentions that the reentrainment of particles due to rapping can be eliminated with the wet ESP approach. There is another way in which humidity appears to help eliminate the problem of reentrainment. A study by Ibrahim et al. in 2004 discovered that increased relative humidity increases the force necessary to pull the particle from the collection plate back into suspension in the air. ${ }^{25}$ Most likely, this effect is caused by adhesion in which water acts like glue to keep the particle attached to the 
collection plate. Ibrahim et al. discovered that for relative humidity below $30 \%$ there is no real effect, but for relative humidity between $30 \%$ and $70 \%$, there is a sharp decrease in the number of particles reentrained for other system parameters held constant. The reentrainment rate appears to remain constant for relative humidity above $70 \%$.

A report by Li and Wen from 2003 yields interesting results that complement the results listed above. ${ }^{26} \mathrm{Li}$ and Wen found that for an ESP designed to remove microorganisms from the air, an increase in relative humidity from $65 \%$ to $85 \%$ resulted in a significant increase in collection efficiency for several flow rates. They credited this increase in efficiency to a, "....higher corona current, observed at higher relative humidity at the same voltage." This is consistent with the idea that humidity reduces resistivity. For a constant voltage, a decrease in resistance should produce an increase in current. This may imply that higher corona voltages can be applied in humid conditions without causing a sparkover.

One group decided to take the positive effects of water in the ESP to the limit with a new design. ${ }^{27}$ This design involved spraying mists from small holes in a hollow corona wire. This idea makes sure that water protects the corona wire and the ground plate from particle build-up which wards off back corona and keeps the particle charging efficient. Thus rapping is not necessary and the collector can still be run continuously. The charged mist is reported to help with agglomeration and to increase collection efficiency for particles of all sizes.

If one does not wish to use water to limit the effects of back corona because of the excess bulk it implies (carrying the water supply with the detector), then other materials with low electrical resisitivities can be used in the corona charging region. This has been demonstrated in at least one instance by researchers at Argonne National Laboratory. ${ }^{28}$ Huang and Gorski showed that $\mathrm{SO}_{3}$ in fly ash effluent can decrease the resisitivity of the fly ash, which keeps the corona current high and thus keeps the charging efficient.

Paris et al. presented experimental results that seem to show that relative humidity causes a high rate of agglomeration in the presence of a corona wire. ${ }^{29}$ For particles with sizes in the range of 1-250 nanometers, they measured particle number concentration and size distribution for a constant corona voltage. They report that for a relative humidity of $58 \%$ the particle number concentration is higher and the average particle diameter is higher than for a relative humidity of $3 \%$. They attribute this to agglomeration of particles. The agglomeration of particles with sizes below the detection limit causes them to grow to sizes within the detection limit, thus increasing the number density. Agglomeration also accounts for the increase in the average particle size.

Surface roughness of the collecting electrode does appear to have a significant effect on collector efficiency. Cheng et al. report that increasing surface roughness significantly reduces the pull-off force. ${ }^{30}$ They claim that it has long been realized that surface roughness significantly reduces adhesion and that a model that does not take into consideration surface roughness underestimates the microparticle detachment rate. They claim that if the standard deviation in the height of asperities is 17 angstroms, then the pull-off force will only be $1 \%$ as large as the pull-off force for a smooth surface. Ibrahim et al. support this claim. ${ }^{31}$ They claim that the mechanism by which particles are detached from a surface is rolling caused by shear from the air. If the surface to which the particle is adhered is rough, or the surface of the particle itself is rough, then the 
contact area between the particle and the surface will be smaller than if the two surfaces were smooth. Ibrahim et al. cite Kim et al. ${ }^{32}$ as claiming, "...a surface roughness of only $45 \AA$ is sufficient to reduce the pull-off force to a very small fraction of its value for smooth surface," and Soltani ${ }^{33}$ as stating, "...for hard elastic materials that have a surface roughness of the order of the atomic scale can reduce adhesion significantly." Johnson presents mathematical descriptions of adhesion of particles to surfaces for different properties. ${ }^{34} \mathrm{He}$ claims that roughness (defined as the dispersion of asperity heights) causes the particle not to be held by adhesive junctions of asperities of varying heights. He claims that, "Significant adhesion can be achieved with compliant solids like rubber..." Hinds presents an empirical expression from $\operatorname{Corn}^{35}$ (Equation A-18) for hard materials and clean surfaces. ${ }^{1(\mathrm{pp} .}{ }^{144)}$ In Equation $\mathrm{A}-18, \mathrm{~d}$ is the particle diameter in meters and \%R.H. is the relative humidity in percent.

$$
F_{a d h}=0.063 d[1+0.009(\% \text { R.H.) }]
$$

Equation A-18

Equation A-18 makes apparent the importance of surface roughness and relative humidity in particle adhesion. The surface area of the particle that can stick to the collecting surface is directly related to the particle size through the particle diameter; the relative humidity is expressed in the equation directly. Hinds also states that the humidity will increase adhesion by water vapor adhering to the particle and the collection surface and creating a liquid that fills in surface fractures which increases contact area. $^{1(\text { pp. } 143)} \mathrm{He}$ also states that, "Coating surfaces with oil or grease increases the adhesion energy, the deformation, and the dissipative energy and greatly reduces the problem of bounce." 1 (pp. 147) Here, bounce is the problem of a particle striking a collection surface and bouncing off instead of adhering. The probability of bounce occurring is given by Equation A-19 where KE is the kinetic energy of the particle. The recommendation of an oil or grease is equivalent to the use of rubber as suggested by Johnson. The more a surface can deform, the more surface area can contact another surface in order to contribute to adhesion.

$$
P_{b}=1-0.000224(K E)^{-0.233}
$$

Equation A-19

A paper by Quon et al. shows that the loss of adhesion strength due to surface roughness can be countered by humidity. ${ }^{36}$ They claim that the smoother the surfaces, the more adhesion there will be. In addition, for smooth surfaces, a relative humidity greater than $80 \%$ causes "stiction" (unusually high adhesion and friction forces) between microsurfaces. For rough surfaces, they state, "Unlike the situation of contact between smooth surfaces, here adhesion can be acutely sensitive to changes in relative humidity. In fact, it is common to see a sharp increase in adhesion at some distinguishable humidity level." In addition, "....adhesion at rough interfaces would be significantly increased when the thickness of adsorbed liquid becomes comparable to the height of asperities. More recent studies find, indeed, that stiction is a maximum when the thickness of liquid films equals the head/disk gap and that stiction at the head/disk interface becomes significant when the thickness of liquid is close to a critical thickness which is related to the magnitude of surface roughness." They conclude that, "The strength of adhesive contact between rough solids can be significantly increased in humid conditions," and that, "Adhesion between these nearly smooth surfaces is not controlled by any one factor - roughness, surface chemistry, and relative humidity all play important, and interrelated, roles." (This suggests there is an optimum mist/film for water in the ESP.) 
Finally, there are those who claim that the corona wire size, shape, separation, current, etc. are vital aspects of the ESP design. Jedrusik et al. ran experiments where different corona shapes were tested to determine how efficiently they drove particles to the collection electrodes. ${ }^{37}$ The corona shapes that they tested were the barbed plate, the wire, the barbed tube, and the spiked band. They concluded that, "The results of research on the influence of the electrode geometry on the migration velocity, as presented here and carried out using the ESP model, suggest that the barbed tube is the most effective and useful corona electrode in respect of effective transport of solid particles towards the collection electrodes. This would support the latest trends towards the widespread application of that type of electrode to ESPs." Miller et al. performed an experiment in which they varied the parameters of a barbed tube to determine the optimum shape. ${ }^{38}$ They concluded that there was an optimum barb separation distance, corona wire separation distance, and optimum corona current. They stated, "For example, the highest efficiency appeared with discharge electrode distances equal to or smaller than half the gap width." Also, "The favorable settings did not necessarily show high total currents, but always exhibited low currents per barb or per discharge electrode. Therefore, the optimum geometric settings result in uniform current density distributions with low current amplitudes at the collecting plates." They go on to say that perhaps back corona is minimized with their small current density. Chang and Bai performed an experiment where they determined that corona geometry effects change with operation indexes. ${ }^{39}$ They claim that if the electric field strength is kept constant, then a decrease in the corona wire diameter will result in a higher collection efficiency. However, if the average current density at the collection plates or the corona power ratio is kept constant, then larger corona wire diameters result in higher collection efficiencies. Also, they claim that for a given electric field, optimal wire-to-wire and plate-to-plate separations can be found. However, if the current density at the plates or the corona power ratio is kept constant, then higher collection efficiencies are always obtained with decreases in the wire-to-wire distance and the plate-to-plate distance. 


\section{Summary of Results of Literature Review:}

- Collection efficiencies for ESP do not follow the Deutsch-Anderson model as claimed in Hinds. Instead, the particle distribution is not homogenous due to finite mixing, resuspension, and convective diffusion. Thus, collection efficiencies are much higher than predicted by the Deutsch-Anderson model. This means that a decrease in flow rate or an increase in collection area might have a greater impact than a logarithmic relationship. These parameters should be more thoroughly investigated.

- The deposition of high resistivity particles on the ground plates and the corona wire in the charger can lead to a phenomenon known as back-corona. This phenomenon can result in a decrease in charging efficiency up to $60 \%$. Back corona can be avoided by decreasing the resistivity of the aerosol with water (humidity) or other conducting materials. Sheath flow and alternating polarity are two other methods to control back corona.

- Increased relative humidity increases particle adhesion, which decreases the rate of resuspension. The positive effect of humidity countering resuspension is claimed to reach its maximum at a relative humidity around $70 \%$ and is nonexistent for a relative humidity below $30 \%$.

- Collection efficiency apparently increases with increasing corona voltage. However, high corona voltages can lead to back corona and sparkover.

- The presence of water (humidity) increases the ion current in the charger due to a higher conductivity. This means that a sparkover will occur at a higher voltage. Thus, higher charger voltages are possible in the presence of water.

- Corona wire thickness can affect the collection efficiency. The general consensus is that a smaller corona wire thickness will provide a higher collection efficiency.

- The distance from the corona wire to the ground plate in the charger can affect the collection efficiency. The general trend is that the smaller the distance from the corona wire to the ground plates, the better the collection efficiency. However, some studies report higher than expected efficiency for higher wire-plate separations. These results are known as non-Deutschian phenomena.

- The distance between corona wires must be optimized. If they are too far away, they will not provide optimum charging conditions (not enough ions); if they are too close, their electric fields will begin to resemble homogenous fields instead of the highly discontinuous geometry necessary to form a corona.

- Corona geometry can affect the charging efficiency. In particular, barbs on the wire or tube seem to increase charging efficiency. 


\section{Synopsis:}

- Collection models (progressively more accurate): All models listed in [2]

o Deutsch-Anderson Model: $\eta_{D-A}=1-\exp \left(-\frac{V_{T E} A_{c}}{Q}\right)$

o Cooperman Model: $\eta_{\text {Coo }}=1-\exp \left[\frac{U L}{2 D}-\sqrt{\left\{\left(\frac{U L}{2 D}\right)^{2}+(1-R) \operatorname{Pe}\left(\frac{L}{W}\right)^{2}\right\}}\right]$

o Leonard Model: $\eta_{\text {Leo }}=1-\int_{0}^{1} P\left(\frac{\xi-D e}{\sqrt{2 D e / P e}}\right) d \xi$

o Zhibin Model: $\eta_{Z h i}=1-\sqrt{\frac{P e}{4 \pi D e}} \int_{0}^{1} \exp \left[-\frac{P e}{4 D e}(\xi-D e)^{2}\right] d \xi$, where

$$
P(z)=\frac{1}{\sqrt{2 \pi}} \int_{-\infty}^{z} \exp \left(-\frac{B^{2}}{2}\right) d B
$$

- The main charging obstacle is the formation of back corona

o The phenomenon of back corona was described thoroughly in 1979 in two articles $[16,17]$. It is essentially the formation of a corona of polarity opposite that of the corona wire on the ground plate. It is caused by the build-up of a layer of high-resistivity material on the ground plate and the corona wire.

o Kim and Lee [2] noticed in 1999 that the collection efficiency of a collector declined with time and attributed this to back corona through the deposition of particles on the ground plate in the charger. They suggested forms of mechanical rapping such as a vibrator or a hammer to control the build up of particles in the charger prevent back corona.

o Chen and Pui [18] in 1999 developed a particle charger with sheath flow to prevent the build-up of a particle layer in the charger.

o Kruis and Fissan [19] in 2001 developed a charger that used alternating polarities to keep the charged particles in the charging volume and off the charger electrodes.

o In 1981, Sekar [22] noted that by increasing the relative humidity in the charger, the effects of back corona can be nearly eliminated. He noticed a significant reduction in back corona when the relative humidity was changed from $10 \%$ to $24 \%$.

o In 1999, Chang and Bai reported that back corona would cause the ESP efficiency to decline with time. They noted that this effect could be eliminated by "conditioning" the flow with a water mist.

o The EPA-CICA Air Pollution Control Technology Fact Sheet for the Wire-Plate Type Wet Electrostatic Precipitator [24] states that, "Because of the high humidity in a wet ESP, the resistivity of particles is lowered, eliminating the back corona condition."

- Increasing Adhesion

o A study by Ibrahim et al. in 2004 [25] discovered that increased humidity increased the force necessary to pull a particle from a collection plate back into suspension in the air. They discovered that there was no adhesion benefit for relative humidities below $30 \%$. However, the adhesion force was enhanced for relative humidities between $30 \%$ and $70 \%$. 
o In 2003, Li and Wen [26] found that for an ESP designed to remove microorganisms from the air, an increase in relative humidity from $65 \%$ to $85 \%$ resulted in a significant increase in collection efficiency for several flow rates.

o Cheng et al. [30] report that increasing surface roughness significantly reduces the pull-off force necessary to remove a particle. The claim that roughness with a standard deviation in the height of asperities of $17 \AA$ decreases the necessary pull-off force to $1 \%$ of its value for smooth surfaces.

o Kim et al. [32] cited in Ibrahim et al. [31] claim that, "...a surface roughness of only $45 \AA$ is sufficient to reduce the pull off force to a very small fraction of its value for smooth surface.

o Quon et al. [36] claim that loss of adhesion strength do to surface roughness can be countered by humidity. They claim for smooth surfaces, a relative humidity greater than $80 \%$ causes "stiction" (unusually high adhesion and friction forces) between microsurfaces.

- Charger Design

o Jedrusik et al. [37] experimented with different corona designs and concluded that, "The results of research on the influence of the electrode geometry on the migration velocity, as presented here and carried out using the ESP model, suggest that the barbed tube is the most effective and useful corona electrode in respect of effective transport of solid particles towards the collection electrodes."

o Miller et al. [38] performed experiments with varied shapes of the barbed tube. They concluded optimal separations for barbs, tubes, and plates.

o Chang and Bai [39] similarly conducted experiments for different corona geometries and parameters. They found different results for constant voltage than for constant corona power.

- Other

o Paris et al. [29] claim that particle agglomeration in a corona charger is greatly enhanced for nanoparticles if the relative humidity is increased from $3 \%$ to $58 \%$.

o Xu et al. [27] developed an ESP that has a hollow corona wire from which a water mist is sprayed. This design is reported to increase collection efficiency for all particle sizes and prevents back corona.

\section{References}

1. William C. Hinds, "Aerosol Technology Properties, Behavior, and Measurement of Airborne Particles," $2^{\text {nd }}$ Edition, John Wiley \& Sons, Inc., 1999.

2. S.H. Kim and K.W. Lee, "Experimental Study of Electrostatic Precipitator Performance and Comparison with Existing Theoretical Prediction Models," J. Electrostatics Vol. 48, No. 1, pp. 3-25, November 1999.

3. G. Cooperman, "A New Theory of Precipitator Efficiency," Atmos. Environ. 5(1971) 541-551.

4. G.L. Leonard, M. Mitchner, S.A. Self, "Experimental Study of the Effect of Turbulent Diffusion on Precipitator Efficiency," J. Aerosol Sci. 13 (1982) 271-284.

5. Z. Zhibin, Z. Guoquan, "New Model of Electrostatic Precipitation Efficiency Accounting for Turbulent Mixing," J. Aerosol Sci. 23 (2) (1992) 115-121.

6. Zhang Xiangrong, Wang Lianze, Zhu Keqin, "An Analysis of a Wire-plate Electrostatic Precipitator," J. Aerosol Sci., Vol. 33, (2002) 1595-1600. 
7. S.J. Park and S.S. Kim, "Effects of Particle Space Charge Density and Turbulent Diffusivity on the Performance of Plate-Plate Electrostatic Precipitators," J. Aerosol Sci., Vol. 27, Suppl. 1. pp. S199-S200, 1996.

8. Seok Joo Park, Sang Soo Kim, "Effects of Particle Space Charge and Turbulent Diffusion on Performance of Plate-Plate Electrostatic Precipitators," Journal of Electrostatics, Vol. 45, (1998) 121-137.

9. B.S. Choi, C.A.J. Fletcher, "Computation of Particle Transport in an Electrostatic Precipitator," Journal of Electrostatics, 40\&41 (1997) 413-418.

10. P. Lawless, "Particle Charging Bounds, Symmetry Relations, and an Analytic Charging Rate Model for the Continuum Regime," J. Aerosol Sci. 27 (2) (1996) 191-215.

11. B.A. Tinsley, R.P. Rohrbaugh, M. Hei, "Electroscavenging in Clouds with Broad Droplet Size Distributions and Weak Electrification," Atmospheric Research, 5960 (2001) 115-135.

12. Jeongsoo Suh, Bangwoo Han, Dae Seong Kim, Mansoo Choi, "A Method for Enhanced Charging of Nanoparticles via Condensation Magnification," J. Aerosol Sci. Article in Press.

13. J.M. Clark, S.R. Preston and D. Shakeshaft, "The Design, Modelling and Experimntal Investigation of a New Electrostatic Particle-in-Water Collection System for Sub-Micrometre Particles," J. Aerosol Sci., Vol. 29, Suppl. 1, pp. S1043-S1044.

14. J.C. Barrett and C.F. Clement, "Growth Rates for Liquid Drops," J. Aerosol Sci. 19(2) (1988), 477-487.

15. Niels Jonassen, "Breakdown," Compliance Engineering Online, http://www.cemag.com/archive/02/01/MrStatic.html.

16. Luong C. Thanh, "Back Corona Part I: Its Formation," Journal of Electrostatics, 6 (1979) 139-160.

17. Luong C. Thanh, "Back Corona Part II: Its Effects on Sparkover Voltage," Journal of Electrostatics, 6 (1979) 161-179.

18. Da-Ren Chen and David Y.H. Pui, "A High Efficiency, High Throughput Unipolar Aerosol Charger for Nanoparticles," Journal of Nanoparticle Research, 1 (1999) 115-126.

19. F.E. Kruis and H. Fissan, "Nanoparticle Charging in a Twin Hewitt Charger," Journal of Nanoparticle Research, Volume 3, issue 1, 2001, pp. 39-50.

20. G. Biskos, K. Reavell, N. Collings, "Unipolar Diffusion Charging of Aerosol Particles in the Transition Regime," J. Aerosol Sci., Vol. 36, (2005), 247-265.

21. A. Hernandez-Sierra, F.J. Alguacil, M. Alonso, "Unipolar Charging of Nanometer Aerosol Particles in a Corona lonizer," J. Aerosol Sci. Vol. 34, (2003), 733-745.

22. S. Sekar, "Studies of Corona in Wire-Plane Electrode System With Backdischarges," Journal of Electrostatics, 10 (1981) 211-216.

23. Chung-Liang Chang and Hsunling Bai, "An Experimental Study on the Performance of a Single Discharge Wire-Plate Electrostatic Precipitator with Back Corona," J. Aerosol Sci. Vol. 30, No. 3, pp. 325-340, 1999.

24. Air Pollution Control Technology Fact Sheet, "Wet Electrostatic Precipitator (ESP)- Wire-Plate Type, EPA-CICA Fact Sheet, EPA-452/F-03-030.

25. A.H. Ibrahim, P.F. Dunn, R.M. Brach, "Microparticle Detachment from Surfaces Exposed to Turbulent Air Flow: Effects of Flow and Particle Deposition Characteristics," J. Aerosol Sci. Vol. 35, pp. 805-821, 2004.

26. Chih-Shan $\mathrm{Li}$ and Yi-Mien Wen, "Control Effectiveness of Electrostatic Precipitation on Airborne Microorganisms," Vol. 37, pp. 933-938, 2003. 
27. Dexuan Xu, Jie Li, Yan Wu, Linhui Wang, Dawei Sun, Zhongyang Liu, Yanbin Zhang, "Discharge Characteristics and Applications for Electrostatic Precipitation of Direct Current: Corona with Spraying Discharge Electrodes," Journal of Electrostatics, 57 (2003) 217-224.

28. Hann Shenn Huang and Anthony J. Gorski, "System for Improving Electrostatic Precipitator Performance by Plasma Reactor Conversion of $\mathrm{SO}_{2}$ to $\mathrm{SO}_{3}$," PATENTS-US-A8937170, Dec. 1, 1997.

29. P. Paris, A. Mirme, M. Laan, "Study of Corona Discharge Aerosol With and Electrical Aerosol Spectrometer," J. Aerosol Sci. Vol. 29, Suppl. 1, pp. S845S846, 1998.

30. W. Cheng, P.F. Dunn, R.M. Brach, "Surface Roughness Effects on Microparticle Adhesion," The Journal of Adhesion, Vol. 78, (2002), pp 929-965.

31. A.H. Ibrahim, P.F. Dunn, R.M. Brach, "Microparticle Detachment from Surfaces Exposed to Turbulent Air Flow: Controlled Experiments and Modeling," J. Aerosol Sci. Vol. 34, (2003) 765-782.

32. Kim, H., Rockfold, L., and Russell, T. (1999), "Adhesion to Rough Surfaces," American Physical Society Centennial Meeting Program, Atlanta, GA.

33. Soltani, M., "Mechanisms of Particle Removal Due to Turbulent Flow or Substrate Acceleration. Masters Thesis, Clarkson University, 1993.

34. K.L. Johnson, "Mechanics of Adhesion," Tribology International, Vol. 31, No. 8, pp. 413-418, 1998.

35. M. Corn, "The Adhesion of Solid Particles to Surface, II," J. Air Pol. Control Assoc., 11, 566-584 (1961).

36. R.A. Quon, A. Ulman, and T.K. Vanderlick, "Impact of Humidity on Adhesion between Rough Surfaces," Langmuir, Vol. 16, pp. 8912-8916, 2000.

37. Maria Jedrusik, Juliusz B. Gajewski, Arkadiusz J. Swierczok, "Effect of the Particle Diameter and Corona Electrode Geometry on the Particle Migration Velocity in Electrostatic Precipitators," Journal of Electrostatics, 51-52, (2001) 245-251.

38. J. Miller, B. Hoferer, A.J. Schwab, "The Impact of Corona Electrode Configuration on Electrostatic Precipitator Performance," Journal of Electrostatics, 44 (1998) 67-75.

39. Chung-Liang Chang and Hsunling Bai, "Effects of Some Geometric Parameters on the Electrostatic Precipitator Efficiency at Different Operations Indexes," Aerosol Science and Technology, 33:228-238 (2000). 